University of Nebraska - Lincoln

DigitalCommons@University of Nebraska - Lincoln

Reducing error and measurement time in impedance spectroscopy using model based optimal experimental design

\author{
Francesco Ciucci \\ University of Heidelberg, Heidelberg, Germany \\ Thomas Carraro \\ University of Heidelberg, Heidelberg, Germany \\ William Chueh \\ Sandia National Laboratories, Livermore, CA \\ Wei Lai \\ Michigan State University, East Lansing, MI
}

Follow this and additional works at: https://digitalcommons.unl.edu/usdoepub

Part of the Bioresource and Agricultural Engineering Commons

Ciucci, Francesco; Carraro, Thomas; Chueh, William; and Lai, Wei, "Reducing error and measurement time in impedance spectroscopy using model based optimal experimental design" (2011). US Department of Energy Publications. 107.

https://digitalcommons.unl.edu/usdoepub/107

This Article is brought to you for free and open access by the U.S. Department of Energy at DigitalCommons@University of Nebraska - Lincoln. It has been accepted for inclusion in US Department of Energy Publications by an authorized administrator of DigitalCommons@University of Nebraska - Lincoln. 


\title{
Reducing error and measurement time in impedance spectroscopy using model based optimal experimental design
}

\author{
Francesco Ciucci ${ }^{a, *}$, Thomas Carraro ${ }^{b}$, William C. Chueh ${ }^{c}$, Wei Lai ${ }^{d}$

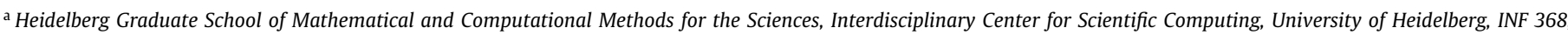 \\ D-69120 Heidelberg, Germany \\ ${ }^{\mathrm{b}}$ Institute of Applied Mathematics, University of Heidelberg, INF 294, D-69120 Heidelberg, Germany \\ c Materials Physics Department, Sandia National Laboratories, Livermore, CA 94551, USA \\ d Department of Chemical Engineering and Materials Science, Michigan State University, East Lansing, MI 48824, USA
}

\section{A R T I C L E I N F O}

\section{Article history:}

Received 1 November 2010

Received in revised form 24 February 2011

Accepted 24 February 2011

Available online 25 March 2011

\section{Keywords:}

Mixed ionic and electronic conductor Equivalent circuits

Identification of physical parameters

Frequency response analysis

\begin{abstract}
A B S T R A C T
In this work we introduce several novel tools for the reduction of errors in parameters estimated with electrochemical impedance spectroscopy experiments. An optimization strategy is developed that minimizes an estimate of the errors on the parameters while bounding the experimental time. The approach is also used to reduce experimental time while keeping a bound on the parameter errors. This feature is particularly critical in systems changing significantly within the experimental time. The paper uses a fuel cell electrode model to test this methodology and presents a real time algorithm for coupling experiment with the parameter estimation and experimental optimization.
\end{abstract}

(c) 2011 Elsevier Ltd. All rights reserved.

\section{Introduction}

Electrochemical impedance spectroscopy (EIS) is a powerful technique used for more than a century [1]. It has been subject of numerous reviews and textbooks [2-4]. EIS has been used in a wide range of topics including sensors analysis and optimization [5], study of dye-sensitized solar cells [6], studies of corrosion [7], biological applications [8], characterization of novel nano materials [9], physical chemistry of solids [10,11], lithium ion batteries [12] and fuel cells [13]. Software packages for the analysis of impedance data have also emerged, see [14-16].

The typical goal of an EIS experiment is to gather significant information regarding the physico-chemical phenomena taking place in an electrochemical device. Typically, a broad frequency sweep is performed, with a linear or a logarithmic spacing between maximum and minimum frequency. The measured data is then compared with a mathematical model that describes the system. The model can be purely empirical, being composed of resistors, capacitors, and generalized electrochemical elements, or otherwise it can be based on the physics of the system. One typically compares the model and the experimental data by fitting the model against the data, i.e., by minimizing a functional that measures the dis-

\footnotetext{
* Corresponding author.

E-mail address: francesco@alumni.caltech.edu (F. Ciucci).
}

tance between model and measurement via the complex nonlinear least squares (CNLS) method. It is clear that errors in the measured data propagate to the estimated parameters and the distribution of such errors can be estimated by analytical expressions under the assumption of "small" errors. We shall assume in this work, that the method used for fitting the data is (unweighted) complex nonlinear least-squares and that the design of the experiment can be optimized in such a way that increases the confidence on the parameters that define the model. Thanks to this procedure, values of the relevant physico-chemical the parameters can be obtained with greater confidence or with a faster experiment.

In an optimized EIS experiment one could aim to reduce the uncertainty of the parameters for given errors in the measurements or to decrease the experimental time without a significant increase in uncertainty. This reduction can be achieved systematically by applying optimal experimental design (OED) [17]. OED comprises of statistical and numerical methods that optimizes the design of the experiment so that the physical parameters are obtained with the greatest statistical confidence. In particular, reducing experimental time while maintaining low errors in the estimated parameters could be significant in situations where uncontrolled fluctuations of the experimental conditions, e.g., temperature, reference electrode position, occur through the experiment's duration, in cases where the systems degrades, and in non-equilibrium electrochemical systems such as batteries under bias. It is important to note that time reduction and accuracy are 
often competing objectives. In fact, for a stable system, longer experimental time will yield more accurate results.

In this work we utilize a physics-based model as an example, however, the presented approach is general and can be easily applied to empirical models, those composed of resistors, capacitors, and generalized elements. For example the latter can be obtained by analyzing the impedance spectra with commonly used software packages [14,16].

We shall stress that the models used to interpret and identify the experiment can also be non-unique, meaning that several models can fit experimental data with comparable residuals [4,18-21], and there can be errors in some of the "known" parameters used in the model. Here we disregard those possibilities and assume that the model describes the physical system precisely. Considering possible errors in the model, the OED technique can also be used for model discrimination [22-24], i.e., for comparing different models which give similar results if tested with standard experimental procedures.

Lastly, the paper will be outlined as follows: first we introduce OED (Section 2), second we describe the model of the example system studied (Section 3), third we review the theory of experimental errors in EIS (Section 4), fourth we apply the OED method to improve impedance measurements (Section 5), and finally we present the results of computations on the given example (Section 6 ). To the best of our knowledge, this is the first time that OED techniques are used to optimize impedance measurements in the field of electrochemistry.

\section{Optimization of model-based experiments}

OED has been studied extensively in the field of statistics $[17,25-27]$ and is frequently used in science and engineering [28-33]. OED largely relies on statistical inference [34] and on the sensitivity analysis of the estimated parameters with respect to perturbations of the data. As stated in the introduction the goal of OED is to find the experimental conditions that reduce the effects of measurement inaccuracies on the estimated parameters. In this sense OED makes the parameter identification less sensitive to sources of error in the measurements. For this purpose, the link between the variations of the data and the variations of the estimated parameters has to be determined and optimized. This link is depicted in Fig. 1. In the traditional approach model and experimental data are used to estimate parameters via CNLS. It is important to note that errors in the data and in the estimated parameters are linked. OED directly exploits this connection and "suggests" more favorable conditions for increasing the confidence on the parameters.

One of the key ideas of OED is to use asymptotic nonlinear estimation to approximate the covariance matrix of the estimated parameters. In order to do that one needs to either assume or measure the error structure of the experimental data. In our case relative errors in the amplitude and in the argument of the measured impedance (see Section 4.2) are considered. The uncertainty in the measured data induces uncertainty in the estimated parameters, i.e., after a fit one should give a mean value for each estimated parameter and an estimation of the variances. The latter are usually represented by error bars. A more complete way to describe the uncertainty is the calculation of the covariance matrix, which gives not only error bars (the diagonal terms of the matrix) but also the correlations between the parameters. A large correlation between two parameters means, that if one of them is known with a large uncertainty, then the other could be affected as well. The covariance matrix, as explained in Appendix B.3, defines the so-called confidence region, which under the assumption of "small" normally distributed errors is depicted by an ellipse (in the case there are only two parameters) or an ellipsoid (in the case the parameters are more than two). The region inside the ellipse defines the range of values that the parameters could have due to measurement errors. To have a pictorial view of the ellipsoidal area we refer back to elementary statistics [35], and for completeness we depict in Fig. 2 two Gaussian probability density functions (p.d.f.s) centered around 0 with covariance matrix $\mathbf{V}$ whose analytical expression is p.d.f. $(\boldsymbol{\theta})=1 /(2 \pi \sqrt{\operatorname{det}(\mathbf{V})}) \exp \left(-(1 / 2) \boldsymbol{\theta}^{\mathrm{T}} \mathbf{V}^{-1} \boldsymbol{\theta}\right)$ and where the parameter vector is $\boldsymbol{\theta}=\left[\begin{array}{l}\theta_{1} \\ \theta_{2}\end{array}\right]$. In the top row we show the p.d.f. with $\mathbf{V}=\left[\begin{array}{ll}1 & 0 \\ 0 & 1\end{array}\right]$, in the bottom panels we depict instead the p.d.f. with $\mathbf{V}=\left[\begin{array}{ll}0.505 & 0.495 \\ 0.495 & 0.505\end{array}\right]$. While the former distribution is circularly symmetric, the latter is elongated and preferentially oriented in the direction $[1,1]^{\mathrm{T}}$. By drawing points from each of the distributions one obtains that most points lie inside the circle in the first case and an elongated ellipse in the second case. Both curves are defined as the points $\boldsymbol{\theta}$ for which $\boldsymbol{\theta}^{\mathrm{T}} \mathbf{V}^{-1} \boldsymbol{\theta}=K$ where $K=9 .{ }^{1}$ One typical goal of OED is to modify the confidence region. Fig. 3 shows schematically the effect of applying OED in case of two parameters $\left(\theta_{1}, \theta_{2}\right)$, where $\hat{\theta}=\left(\hat{\theta}_{1}, \hat{\theta}_{2}\right)$ is the vector of the parameters estimated via CNLS.

In optimizing EIS, one could use OED to select the frequencies that lead to greater parameter confidence (see Section 6.1). Another strategy could determine the "best" sample geometry for the identification of the parameters in the system under study (see Section 6.2). This is achieved by connecting the error in the measurements with the error on the parameters Fig. 1 and subsequently by modifying the experimental design $\xi$ so that the confidence on the estimated parameters in increased, Fig. 3. Depending on the experiment, OED can improve in different ways the results of the nonlinear estimation procedure. Specifically OED can

- decrease the volume of the confidence region;

- decrease the correlation between the parameters;

- optimize the experimental conditions, for example, by decreasing the overall time of the experiment or allowing to take measurements at more convenient conditions.

The use of the covariance matrix $\mathbf{V}$ of the parameters estimates to define the confidence region has been proven useful in many applications that use nonlinear models [31,36,37]. However, the direct determination of $\mathbf{V}$, for example via a Monte Carlo method, would be computationally taxing even for a small number of parameters. An asymptotic estimate $\mathbf{V}_{\text {asympt }}$ of matrix $\mathbf{V}$, see Appendix C, has a clear computation advantage over Monte Carlo strategies because the number of computations necessary to achieve an informative result regarding the covariance matrix is greatly reduced. In addition, the derivatives of functions of $\mathbf{V}_{\text {asympt }}$ can be easily computed numerically, for example via finite differencing or automatic differentiation [38], and can be used in existing optimization algorithms to solve an OED problem. We note that this optimization is rather complex since $\mathbf{V}_{\text {asympt }}$ is a nonlinear function of $\boldsymbol{\theta}$, of the measurement error structure, and it depends on the method used for fitting the data, for details see Appendix C.15 in Appendix C.

The design of an optimal experiment for improving parameter precision reduces then to minimizing a functional of the asymptotic

\footnotetext{
${ }^{1} K=9$ corresponds to assuming that the data will likely fall within 3 standard deviations of the average value.
} 


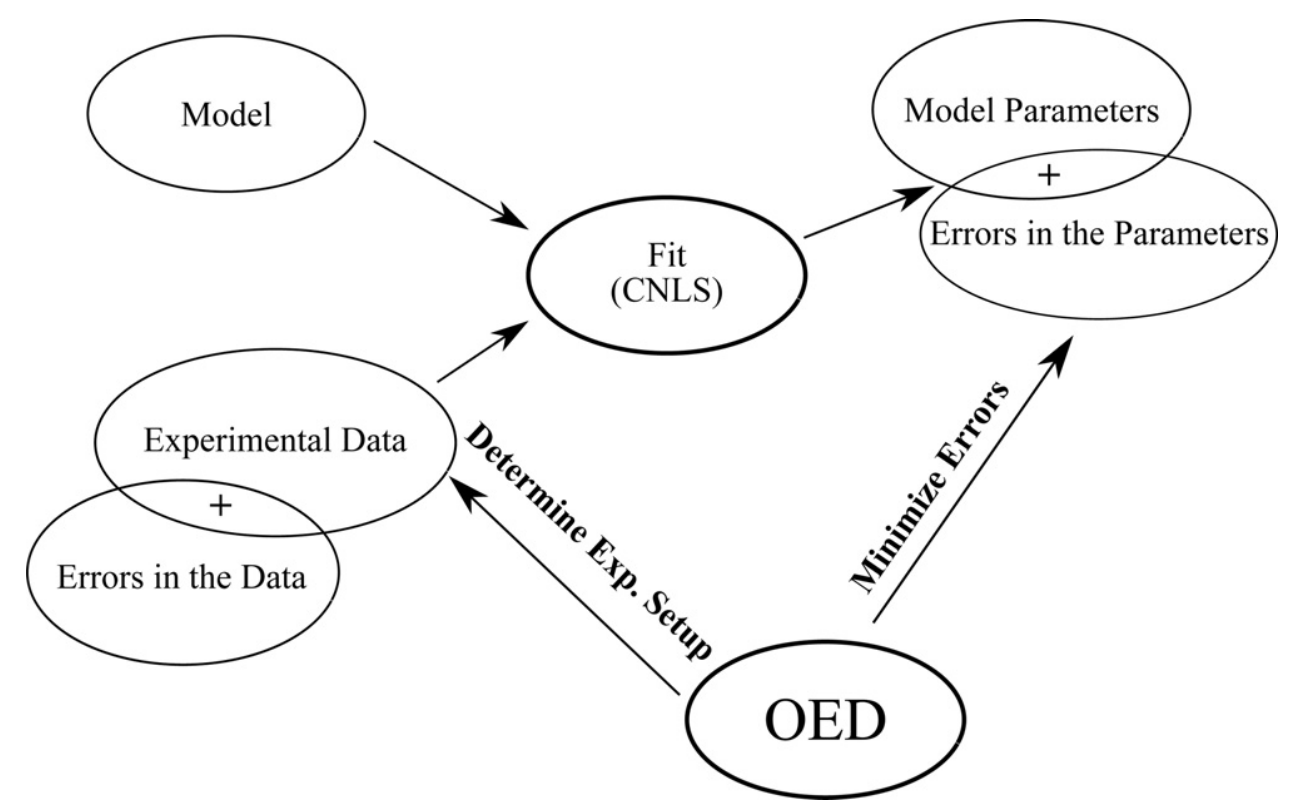

Fig. 1. Sketch of the OED approach that couples measurement errors and estimated parameter confidence and aims at modifying the experimental conditions to improve the latter.
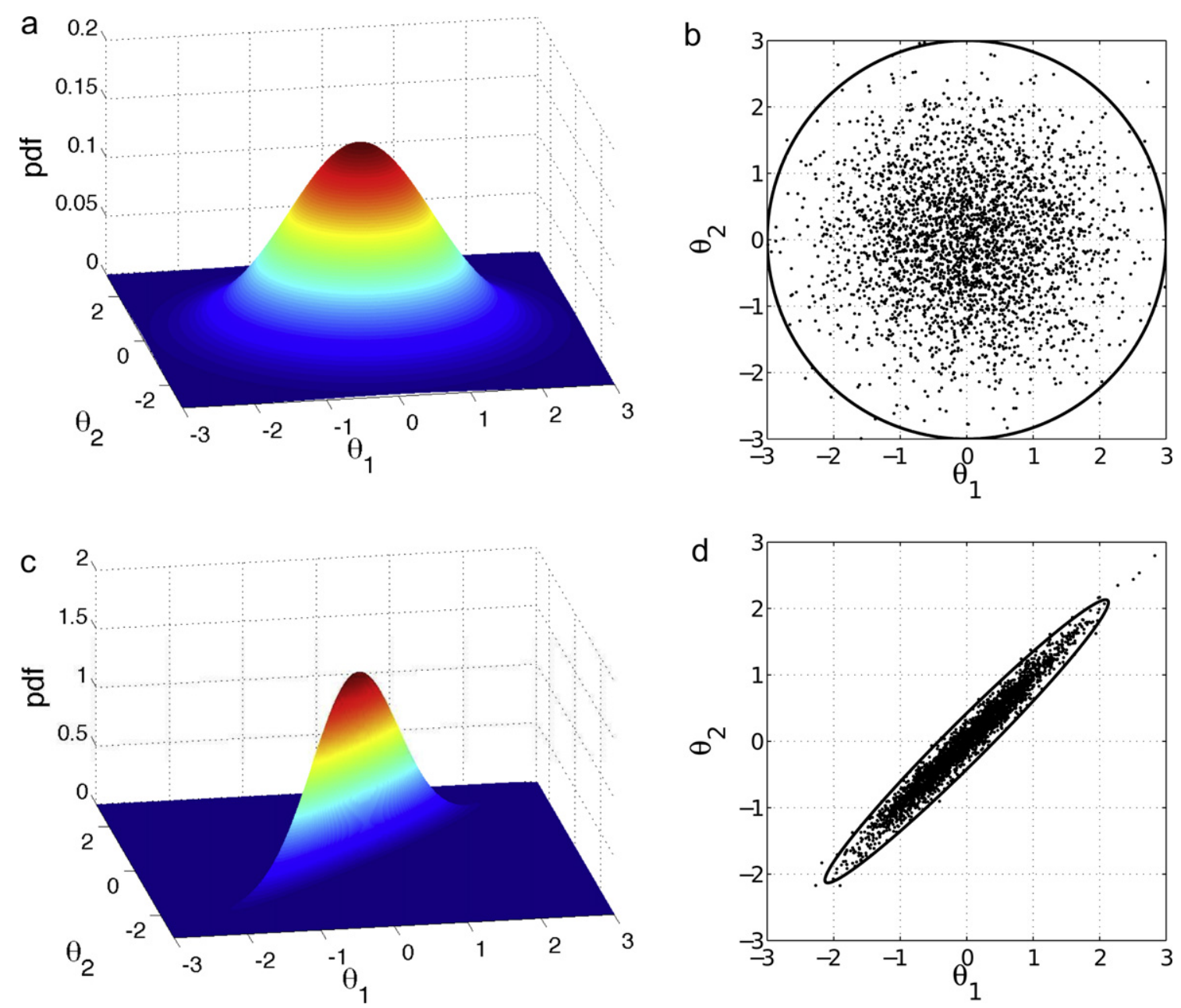

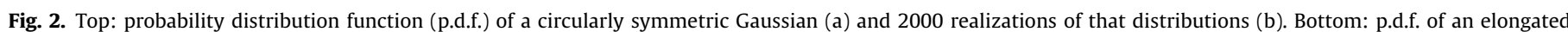

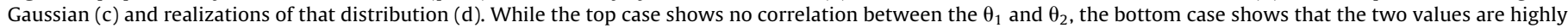
correlated. The vast majority of the realizations are contained in the set $\boldsymbol{\theta}^{\mathrm{T}} \mathbf{V}^{-1} \boldsymbol{\theta} \leq K$, in this case we choose $K=9$. 

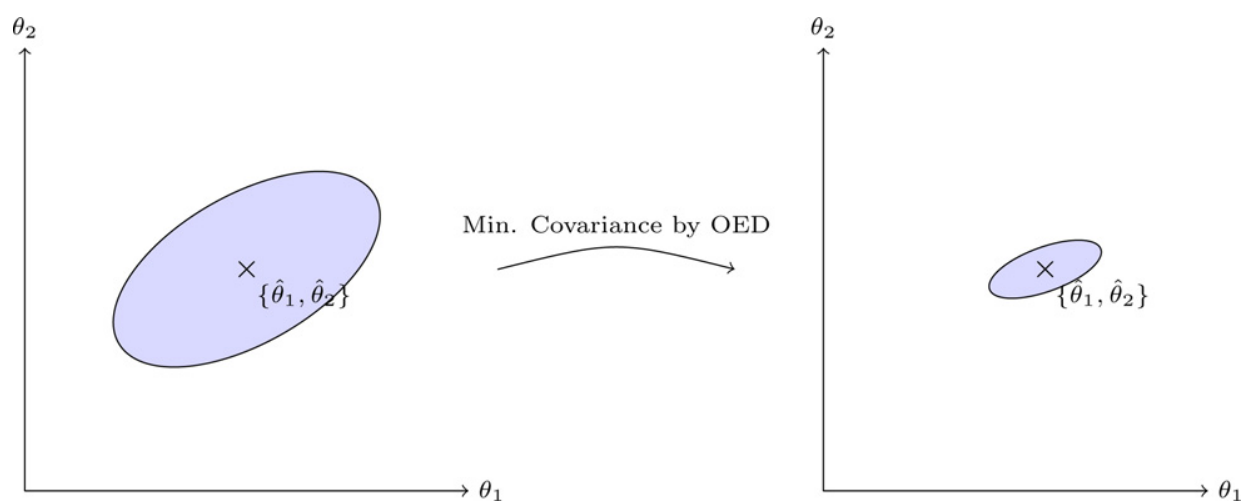

Fig. 3. Reduction of the confidence region by means of OED. The area of the confidence region before the optimization (left) is reduced by OED (right). The linearized confidence region is defined by the parameter values that lie within the ellipsoid defined by $\boldsymbol{\theta}^{T} \mathbf{V}^{-1} \boldsymbol{\theta}=K$, where $\mathbf{V}$ is the covariance matrix, where $K$ is a predefined level and $\boldsymbol{\theta}=\left[\theta_{1}, \theta_{2}, \ldots, \theta_{N}\right]$. The projection of the ellipsoid on the plane $\left(\theta_{i}, \theta_{j}\right)$, such that $i, j=1,2, \ldots, N, i \neq j$, is an ellipse. The bigger this region is, the more inaccurate the estimate will be.

covariance matrix $\mathbf{V}_{\text {asympt }}$ of the estimated parameter set $\hat{\boldsymbol{\theta}}$. The steps one takes in OED can be summarized as follows:

1. define some design variables $\xi$ with which one can control the experiment;

2. derive the asymptotic estimate $\mathbf{V}_{\text {asympt }}$ of the exact matrix of covariances $\mathbf{V}$;

3. set an optimization problem that minimizes the confidence region of the estimated parameters $\hat{\boldsymbol{\theta}}$ using design variables $\boldsymbol{\xi}$.

The latter point be further summarized in the form of the following mathematical problem

$\min _{\boldsymbol{\xi} \in \Xi} \Phi\left(\mathbf{V}_{\text {asympt }}(\boldsymbol{\xi}, \hat{\boldsymbol{\theta}})\right)$

where $\Xi$ is the design space and $\Phi$ is a suitable function of the asymptotic covariance matrix $\mathbf{V}_{\text {asympt }}$. The design variable space $\Xi$ often reduces to a box constraint for the components of the design, as it is the case here, see (8). In this work we will focus solely on reducing the trace of $\mathbf{V}_{\text {asympt }}$, see Appendix B.4, since its minimization corresponds to the overall amelioration of the confidence on the estimated parameters. ${ }^{2}$ We could, however, envision OED approaches that aim at minimizing the error of only one parameter, disregarding its correlations with other parameters. Specifically if one aims at knowing well only the $k$ th parameter $\theta_{k}$, then the goal will be to minimize the matrix element $\left(\mathbf{V}_{\text {asympt }}\right)_{k k}$. For completeness a brief review of the theory of OED is given in Appendix B.

\section{Model}

In this paper, we apply the OED technique to optimize the impedance spectroscopy measurement of solid-state electrochemical cells. Specifically, we have selected the system where a mixed ionic and electronic conductor (samarium doped cerium oxide, or $\mathrm{SDC}$ ) is sandwiched between two porous metal electrodes (Pt). The availability of an experimentally validated physical model [39-42] as well as the number of estimated parameters ( 3 or 4 ) motivates the selection of this model system to test our approach.

The Pt $\mid$ SDC $\mid$ Pt cell is placed in a uniform environment and characterized at open-circuit conditions. For more information on the experimental details, see Lai and Haile [40] and Chueh et al. [42]. Under a chemically reducing environment, SDC conducts both

\footnotetext{
2 The trace of $\mathbf{V}_{\text {asympt }}$ is proportional to the sum of the squares of the semi-axis lengths of the confidence ellipsoid.
}

oxygen vacancies and polarons. We assume that the oxygen vacancies charge-transfer reactions at the solid I gas interface is much slower than the rate of electron transfer. With this assumption [43], the Poisson-Nernst-Planck transport equations with suitable boundary conditions can be transformed into an equivalent circuit (Fig. 4(a)) and the following analytical impedance expression is shown to apply, see $[40,44]$ :

$$
\begin{aligned}
Z(f)= & R_{\infty}+\left(R_{0}-R_{\infty}\right)\left(1+\frac{R_{\text {ion }}+R_{\text {eon }}}{2 R_{\text {ion }}}\right) \\
& \times \frac{\tanh s(f)}{s(f)+\left(\left(R_{\text {ion }}+R_{\text {eon }}\right) / 2 Z_{\text {ion }}^{\perp}\right) \tanh s(f)}
\end{aligned}
$$

where $f$ is the frequency, $R_{\text {ion }}$ and $R_{\text {eon }}$ are the bulk ionic and electronic resistance, $R_{i o n}^{\perp}$ is the electrode resistance, and $s(f)$ is the effective frequency (see Table 1 for additional definitions and Table 2 for the numerical values used). We emphasize that while (2) is expressed in terms of resistances and generalized frequencies, the actual physical parameters we shall investigate $\left(c_{e o n}\right.$, the concentration of electrons, $D_{\text {eon }}$, the electronic diffusivity, $D_{\text {ion }}$, the ion diffusivity and $Z_{i o n}^{\perp}$, the interfacial resistance) can be obtained using Table 1. It has been shown [45] that the electrode resistance is directly related to the porosity of the metal current collector, $r_{A}$ :

$Z_{\text {ion }}^{\perp}=R_{\text {ion }}^{\perp} r_{A}$

Table 1

Definitions of the key terms in the 1D model of the single-slab system of Fig. 4.

\begin{tabular}{lll}
\hline$D_{\text {eon }}$ & $\frac{u_{\text {eon }}}{k_{B} T}$ & Electronic diffusivity \\
$D_{\text {ion }}$ & $\frac{u_{\text {eon }}}{k_{B} T}$ & Vacancy diffusivity \\
$\sigma_{\text {eon }}$ & $\frac{D_{\text {eon } c_{\text {eon }} z_{\text {eon }}^{2} e^{2}}^{k_{B} T}}{\frac{D_{\text {ion }} c_{\text {ion }} z_{\text {ion }}^{2} e^{2}}{k_{B} T}}$ & Electronic conductivity \\
$\sigma_{\text {ion }}$ & $r_{A} R_{\text {ion }}^{\perp}$ & Vacancy conductivity \\
$Z_{\text {ion }}^{\perp}$ & $2 L / \sigma_{\text {eon }}$ & Interfacial resistance \\
$R_{\text {eon }}$ & $2 L / \sigma_{\text {ion }}$ & Electronic bulk resistance \\
$R_{\text {ion }}$ & $\frac{1}{\left(1 / R_{\text {eon }}\right)+\left(1 /\left(R_{\text {ion }}+2 Z_{\text {ion }}^{\perp}\right)\right)}$ & Vacancy bulk resistance \\
$R_{0}$ & $1 /\left(1 / R_{\text {eon }}+1 / R_{\text {ion }}\right)$ & Impedance at $f \rightarrow 0$ \\
$R_{\infty}$ & $\frac{e^{2}}{k_{b} T} L /\left(1 /\left(z_{\text {eon }}^{2} c_{\text {eon }}\right)+1 /\left(z_{\text {ion }}^{2} c_{\text {ion }}\right)\right)$ & Chemical capacitance \\
$C_{\text {chem }}$ & $L^{2} /\left(\left(R_{\text {ion }}+R_{\text {eon }}\right) C_{\text {chem }}\right)$ & Chemical diffusivity \\
$\tilde{D}$ & $\sqrt{\frac{i 2 \pi f L^{2}}{\tilde{D}}}$ & Effective frequency \\
$S$ & &
\end{tabular}



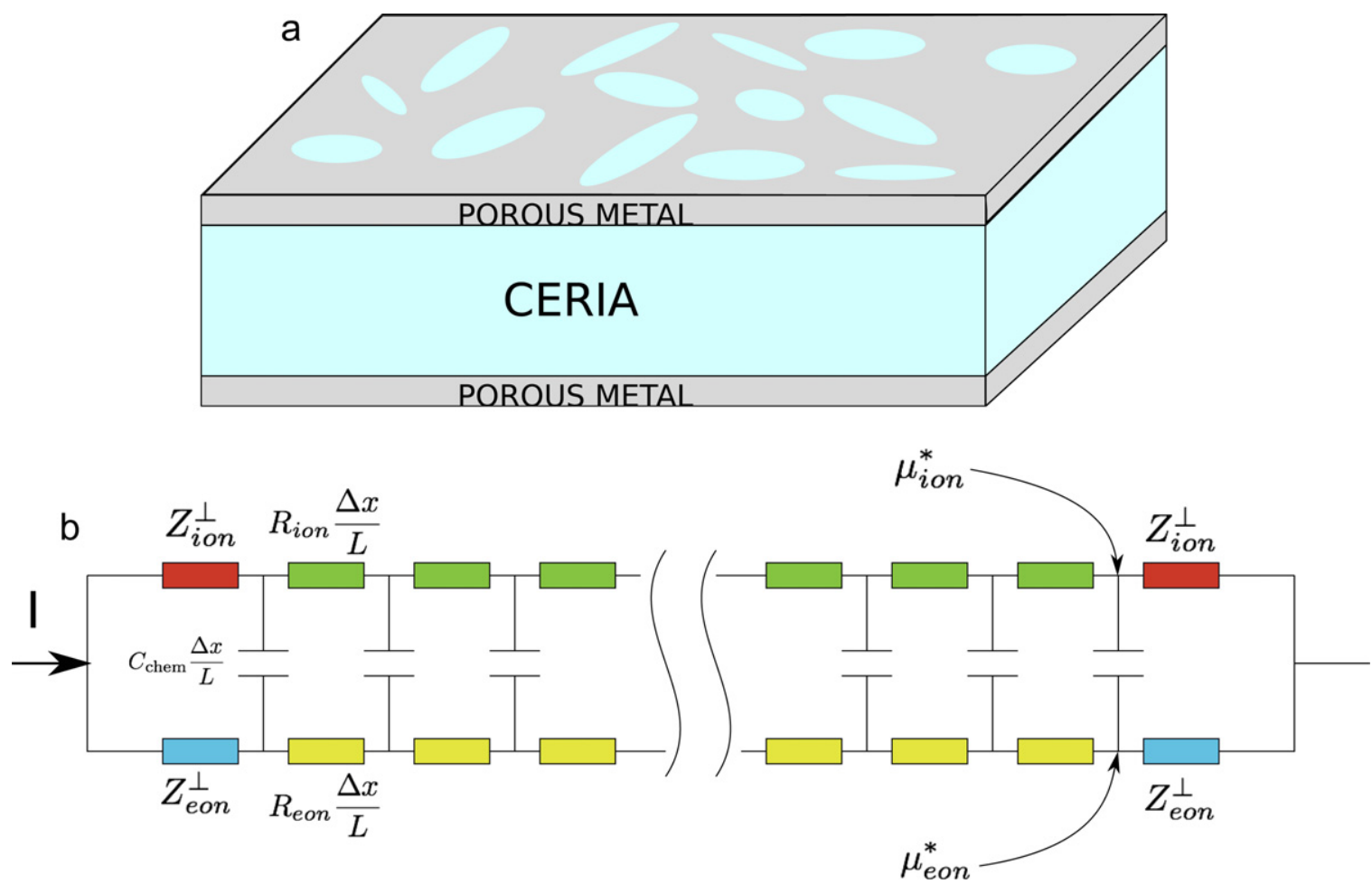

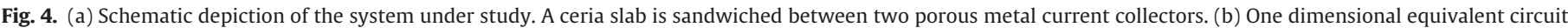

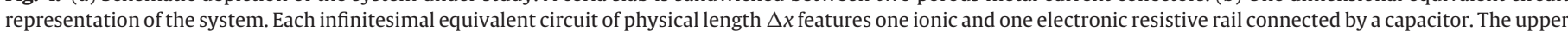

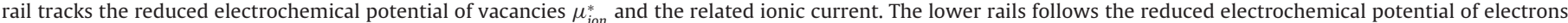
$\mu_{\text {eon }}^{*}$ and the electronic current $[39,40]$.

where $r_{A}=\left(A_{\text {exposed }} / A_{\text {cross section }}\right)$ is the ratio of the exposed ceria area divided by its cross sectional area [41], or in other words the percentage of exposed SDC in the electrode. By definition $0 \leq r_{A} \leq 1$.

It is immediately obvious from (2) and Table 1 that the bulk ionic and electronic resistances ( $R_{\text {ion }}$ and $\left.R_{\text {eon }}\right)$ are strongly correlated terms. Experimentally, $R_{\text {ion }}$ is typically measured independently under different oxygen gas-phase activities which the SDC is an ion-only conductor SDC changes from a mixed ionic and electronic conductor to an ion-only conductor. In Sections 6.1 and 6.2, we will use the independently measured $R_{i o n}$, where we assume the ion concentration is fixed by the high doping level, and optimize the measurement of electron concentration $\left(c_{\text {eon }}\right)$, electronic diffusivity $\left(D_{\text {eon }}\right)$, and electrode resistance $\left(R_{\text {ion }}^{\perp}\right)$. In Section 6.3, we will relax this assumption and, in addition to the three parameters above, we will optimize also the estimation of the ionic diffusivity $\left(D_{i o n}\right)$.

\section{Statistical error in EIS}

The determination of the covariance matrix of the estimated parameters requires information about the underlying error, see

Table 2

Temperature range and material constants used in the simulations.

\begin{tabular}{lll}
\hline$T$ & $650^{\circ} \mathrm{C}$ & Temperature \\
$\bar{c}_{\text {eon }}$ & $1.874 \times 10^{26} \frac{\text { particles }}{\mathrm{m}^{3}}$ & Electron concentration \\
$\bar{c}_{\text {ion }}$ & $1.8738 \times 10^{27 \frac{\text { particles }}{\mathrm{m}^{3}}}$ & Vacancy concentration \\
$\overline{\mathrm{u}}_{\text {eon }}$ & $8.123 \times 10^{-8} \frac{\mathrm{m}^{2}}{\mathrm{sV}}$ & Electron mobility \\
$\overline{\mathrm{u}}_{\text {ion }}$ & $4.936 \times 10^{-9} \frac{\mathrm{m}^{2}}{\mathrm{sV}}$ & Vacancy mobility \\
$\bar{R}_{\text {ion }}^{\perp}$ & $2.16 \Omega \mathrm{cm}^{2}$ & Area specific resistance \\
$r_{A}$ & Varied & Metal porosity \\
$L$ & Varied & Sample thickness \\
\hline
\end{tabular}

Appendix B. In this section we briefly review the literature in the area and we give details of our error implementation.

\subsection{General theory}

Error analysis of EIS experiments has been widely studied over the past 20 years mainly by Orazem and coworkers [46-50], Dygas and Breiter [51], Zoltowski [52-54], Macdonald and Franceschetti [55], Dickinson and Whitfield [56], Horvat-Radosevic et al. [57], and Hilpert [58]. The determination of the error structure plays a pivotal role in the fitting EIS models against measurement data and it is closely linked to the optimal determination of appropriate weights in the least squares functional. Various approaches for mitigating error propagation have emerged over the past 25 years, including modified proportional weighting [59], maximum likelihood theory [60] and multivariate coupled identification of the error structure [51]. The measurement error can be thought as the offset between the measured impedance $Z_{\text {meas }}$ and the model $Z$. It is reasonable then to assume that in order to find the accurate parameter estimate $\hat{\boldsymbol{\theta}}$, the following functional needs to be minimized $[61,62]$ :

$$
\begin{aligned}
\mathbb{S}(\boldsymbol{\theta})= & \sum_{j=1}^{P}\left|Z\left(\boldsymbol{\theta}, f_{j}\right)-Z_{\text {meas }}\left(\boldsymbol{\theta}, f_{j}\right)\right|^{2} \\
= & \sum_{j=1}^{P}\left(\Re Z\left(\boldsymbol{\theta}, f_{j}\right)-\Re Z_{\text {meas }}\left(\boldsymbol{\theta}, f_{j}\right)\right)^{2} \\
& +\sum_{j=1}^{P}\left(\Im Z\left(\boldsymbol{\theta}, f_{j}\right)-\Im Z_{\text {meas }}\left(\boldsymbol{\theta}, f_{j}\right)\right)^{2} .
\end{aligned}
$$

Further details on the fitting procedure are given in Appendix D.1. 


\subsection{Model errors}

As stated above the method relies on the model defined in Section 3. This model depends on the following dimensional parameter array $\boldsymbol{\theta}_{\text {dim }}=\left[c_{\text {eon }}, D_{\text {eon }}, R_{\text {ion }}^{\perp}\right]^{\mathrm{T}}$. Since we shall focus on minimizing relative errors, and not total errors, the parameters will be expressed in dimensionless form defined as $\boldsymbol{\theta}=\left[\theta_{1}, \theta_{2}, \theta_{3}\right]^{\mathrm{T}}$, where

$\theta_{1}=\frac{c_{\text {eon }}}{\overline{\bar{c}}_{\text {eon }}}$

$\theta_{2}=\frac{D_{e o n}}{\bar{D}_{e o n}}$

$\theta_{3}=\frac{R_{i o n}^{\perp}}{\bar{R}_{\text {ion }}^{\perp}}$

One could also estimate a 4th parameter directly from the impedance data. This gives the following "augmented" parameter set $\boldsymbol{\theta}_{\text {augm }}=\left[\boldsymbol{\theta}, \theta_{4}\right]^{\mathrm{T}}$ where

$\theta_{4}=\frac{D_{i o n}}{\bar{D}_{i o n}}$

In Sections 6.1 and 6.2, we assume $\theta_{4}$ is known from an independent experiment. In Section 6.4, we relax this assumption and estimate a total of four parameters.

We briefly analyze the experimental data from the data from Chueh et al. [42]. We compared the data with the model (2) and found the residual to be reasonable well described by:

$$
Z(\boldsymbol{\theta}, f)-Z_{\text {meas }}(f)=\mathcal{N}\left(0, \sigma_{A}^{2}\right) Z(\boldsymbol{\theta}, f)+i \mathcal{N}\left(0, \sigma_{\phi}^{2}\right) \arg (Z(\boldsymbol{\theta}, f)) Z(\boldsymbol{\theta}, f),
$$

where $Z(\boldsymbol{\theta}, f)$ is defined in (2), $\mathcal{N}\left(0, \sigma^{2}\right)$ is a Gaussian stochastic process of zero mean and variance $\sigma$, and where we take $\sigma_{A}=0.5 \%$ and $\sigma_{\phi}=3 \%$. We note that the $\sigma_{A}$ is the relative error on the amplitude of the impedance and $\sigma_{\phi}$ is the relative error on the phase of the measured impedance. This estimation of the error is in accordance with data provided by the impedance spectrometer's manufacturer [63]. Also, the values selected roughly include the inaccuracies from the impedance analyzer and other measurement errors, such as variations of temperature, gas composition, sample thickness, lead length, etc. It is also assumed that the error structure comprises "small" model inaccuracies. For reference, a sample stochastic impedance is compared to the model impedance in Fig. 5.

\section{Application of the OED method to impedance experiments}

The model has the following "tunable" experimental features: the metal current collector porosity $\left(r_{A}\right)$, the sample thickness $(L)$, and the frequency examined with impedance spectroscopy $(\mathbf{f}=$ $\left[f_{1}, \ldots f_{P}\right]^{\mathrm{T}}$, where $\mathbf{f}$ is a vector containing $P$ frequency points). The parameters $r_{A}$ and $L$ can be modified during the set up of the experiment while the $\mathbf{f}$ can be changed "on the fly" as the experiment is performed. We further note that $P$, the number of measurements in the frequency sweep, is not necessarily a preset quantity, The tunable parameters are bounded. Specifically, we note that the frequency points are bounded, with the higher bound given by the frequency in which impedance measurement can be made accurately without parasitic interference, and the lower bound given by the frequency in which the measurement can be made in a reasonable time. We shall assume the following box constraints:

$0.1 \mathrm{~mm} \leq L<1 \mathrm{~mm}$,

$0.1 \leq r_{A} \leq 0.8$

$10^{-3} \mathrm{~Hz}=f_{\min } \leq f_{p} \leq f_{\mathrm{MAX}}=10^{4} \mathrm{~Hz}$.
Lastly we chose that a typical experiment has one of the two objectives:

1. Minimize the experimental time $t_{\exp }=\sum_{j} \max \left(\left(2 / f_{j}\right), 1 \mathrm{~s}\right)^{3}$;

2. Minimize the trace of the asymptotic covariance matrix $\mathbf{V}_{\text {asympt }}$ of the fitted experimental parameters (minimize the sum of all the errors on all estimated parameters).

In our example the design variables $\xi$ can be any subset of the tunable features of the experiment defined above, e.g., $\left[r_{A}, L\right]$, [f] or $\left[f_{Q+1}, f_{Q+2} \ldots, f_{P}\right]$, where the $\left[f_{1}, f_{2}, \ldots, f_{Q}\right](Q<P)$ are fixed.

Thanks to the assumption of small errors, the exact covariance matrix can be estimated by the asymptotic covariance matrix $\mathbf{V}_{\text {asympt }}(\hat{\boldsymbol{\theta}}, \boldsymbol{\xi})$, whose derivation can be found in Appendix C. It is important to note that alternatively the error can be calculated by means of a stochastic simulation. Assuming that an experiment gives results according to the impedance distribution (7), then the method consists on drawing a large number of times the following stochastic process:

$Z_{\text {stoch }}(\boldsymbol{\theta}, f)=Z(\boldsymbol{\theta}, f)\left[1+\mathcal{N}\left(0, \sigma_{A}^{2}\right)+i \mathcal{N}\left(0, \sigma_{\phi}^{2}\right) \arg (Z(\boldsymbol{\theta}, f))\right]$,

for the given frequency sweep and determining for each sweep the set of parameters $\hat{\boldsymbol{\theta}}$ minimizing the least squares functional $\mathbb{S}$, defined as the distance between measured impedance and the model. The covariance matrix $\mathbf{V}_{\text {stoch }}$ is then computed as the covariance of the fitted $\hat{\boldsymbol{\theta}}$, since we chose that $\mathbb{E}\left[\hat{\theta}_{k}\right]=1$ (the $\boldsymbol{\theta}$ is by definition normalized, see (5)), then we can define the stochastic variance as

$\left(\mathbf{V}_{\text {stoch }}\right)_{h k}=\frac{1}{M} \sum_{m=1}^{M}\left(\hat{\theta}_{h}(m)-1\right)\left(\hat{\theta}_{k}(m)-1\right)$,

where $M$ is the total number of stochastic impedances drawn. It is clear that $\mathbf{V}_{\text {stoch }} \rightarrow \mathbf{V}$ in case $M \rightarrow \infty$. We used this approach to compute the values given in Tables 3 and 4 .

\section{Results}

We now examine optimization strategies for error reduction in the model system detailed above. First we analyze optimal selection of frequencies, in the case of 3 parameters $\left(c_{\text {eon }}, D_{\text {eon }}, R_{i o n}^{\perp}\right)$, see Section 5, second we discuss the impact of the geometry on parameter errors, third we consider that the model has one additional parameter $D_{i o n}$, fourth we simulate an experiment in which we select the next frequency in real time on the basis of OED. The computational details of the optimization are given in Appendix D.

\subsection{OED for the distribution of frequencies}

We shall start with the discussion of the confidence region in the case of a frequency sweep vector $\mathbf{f}_{\text {log }}$, which has 50 elements logarithmically spaced between the $f_{\min }$ and $f_{\text {MAX }}$. This frequency placement is commonly used in commercial impedance analyzers [4]. The region is compared to the asymptotic confidence region, for visual simplicity we show only the projection of the ellipsoid Fig. $6 .^{4}$ The ellipsoid projections show that there is good agreement between computational experiments and estimated covariance

\footnotetext{
3 The experiments are run such that they last a minimum $1 \mathrm{~s}$ and cover at least of two cycles.

${ }^{4}$ The ellipsoid is computed as set of $\hat{\boldsymbol{\theta}}$ 's such that $(\hat{\boldsymbol{\theta}}-\overline{\boldsymbol{\theta}})^{\mathrm{T}} \mathbf{V}^{-1}(\hat{\boldsymbol{\theta}}-\overline{\boldsymbol{\theta}})=9$. We recall that given a normally distributed random variable centered around 0 , the probability that a randomly drawn point is outside the interval $[-3 \sigma, 3 \sigma]$ is approximately $0.27 \%$ [35]. In all figures we show only the projections of the ellipsoid.
} 

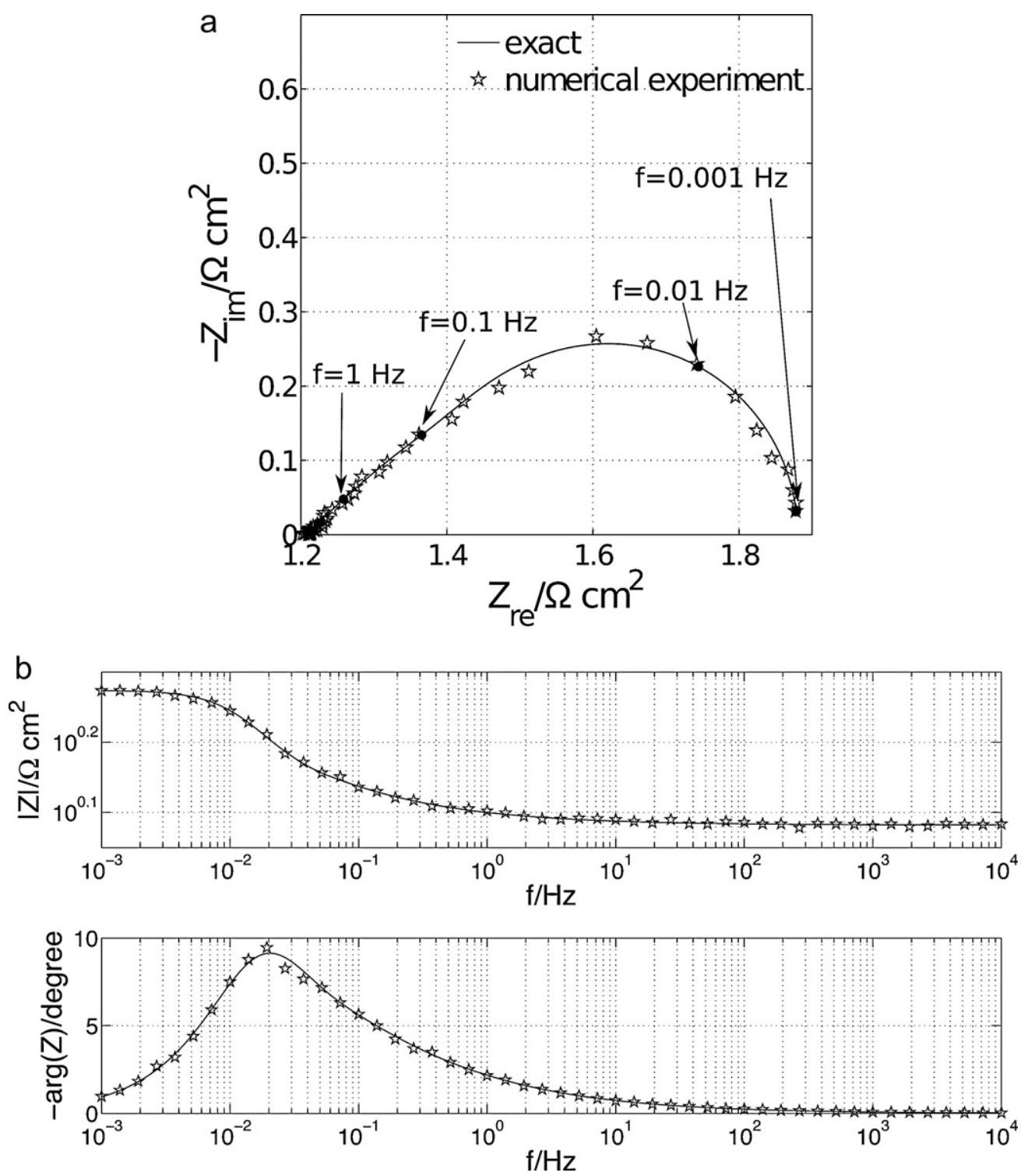

Fig. 5. Stochastic (symbols) versus exact (solid line) impedance as a Nyquist plot (a) and as Bode plots (b).

matrix $\mathbf{V}_{\text {asympt }}$ in the case of relative errors of $0.5 \%$ in the amplitude of $Z$ and $3 \%$ in the argument of $Z$. This confirms that the $\mathbf{V}_{\text {asympt }}$ is indeed a good estimator of the exact $\mathbf{V}$. We note from the top left panel of Fig. 6 that parameters $c_{\text {eon }}$ and $D_{\text {eon }}$ are highly correlated as a sizable error on one parameter relates to a substantial error in the other. The relative errors in the estimated parameters were determined by a Monte Carlo algorithm, and are given by the diagonal elements of $\mathbf{V}_{\text {stoch }}$ for $M=50,000$, see Table 3. It is important to note that in the $\mathbf{f}_{\log }$ was the frequency span used in the experiments of Chueh et al. [42]. In this case two frequency extrema are fixed, $f_{\min }=10^{-3} \mathrm{~Hz}, f_{\mathrm{MAX}}=10^{4} \mathrm{~Hz}$, and the frequencies between them are spanned logarithmically. The experimental time in the case one chooses 50 points in the sweep is $119.3 \mathrm{~min}$ as reported in Table 3.

If we optimize the two ends of the frequency spectrum while maintaining to $50 \mathrm{log}$-spaced sampling points between the two

Table 3

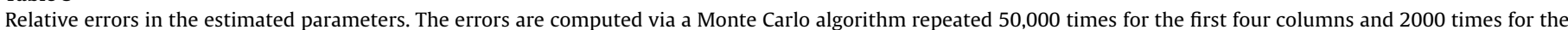

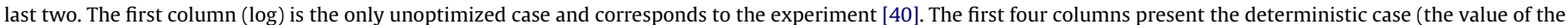

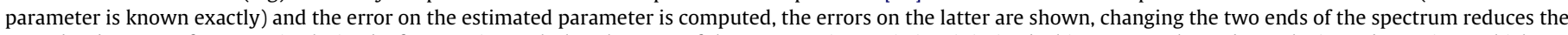

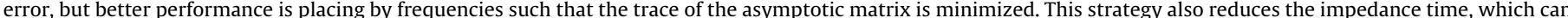

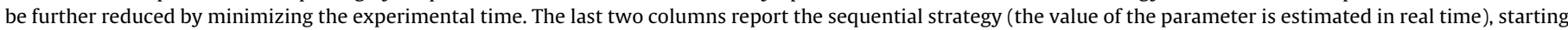
with $P=29$ and going until $P=100$. In the case the trace is minimized, the error is reduced, in the case the trace is minimized the error decreases by a factor 4 .

\begin{tabular}{|c|c|c|c|c|c|c|}
\hline & \multicolumn{4}{|c|}{ Deterministic } & \multicolumn{2}{|l|}{ Sequential } \\
\hline & $\log$ & $\min _{f_{\min }, f_{M A X}} \operatorname{tr}(V)$ & $\min _{\mathrm{f}} \operatorname{tr}(V)$ & $\min _{\mathbf{f}} t_{\exp }$ & $\min _{f_{p+1}} \operatorname{tr}(V)$ & $\min _{f_{p+1}} t_{\exp }$ \\
\hline rel. error $c_{e o n}$ & $4.2 \%$ & $3.2 \%$ & $3.0 \%$ & $3.7 \%$ & $2.6 \%$ & $3.9 \%$ \\
\hline rel. error $D_{\text {eon }}$ & $4.4 \%$ & $3.7 \%$ & $3.4 \%$ & $4.0 \%$ & $2.8 \%$ & $4 \%$ \\
\hline rel. error $D_{\text {eon }}$ & $4.7 \%$ & $4.2 \%$ & $4.4 \%$ & $5.4 \%$ & $3.5 \%$ & $4.8 \%$ \\
\hline$t_{\exp } / \min$ & 119.3 & 92.7 & 63.2 & 28.2 & 115.3 & 36 \\
\hline
\end{tabular}


Table 4

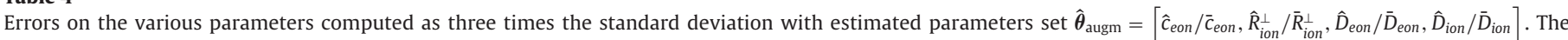

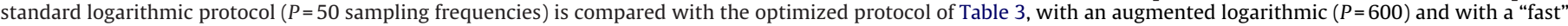
trace minimizing sweep with $P=600$ measurements. The standard deviations are determined by running the stochastic impedance estimation 50,000 times.

\begin{tabular}{|c|c|c|c|c|}
\hline & $\log (P=50)$ & $\min \operatorname{tr}(V)(P=50)$ & $\log (P=600)$ & $\min \operatorname{tr}(V)(P=600)$ \\
\hline rel. error $c_{e o n}$ & $13.5 \%$ & $8.8 \%$ & $3.8 \%$ & $3.4 \%$ \\
\hline rel. error $D_{\text {eon }}$ & $18.2 \%$ & $12.1 \%$ & $5.2 \%$ & $4.4 \%$ \\
\hline rel. error $R_{i o n}^{\perp}$ & $29.3 \%$ & $20 \%$ & $8.1 \%$ & $5.6 \%$ \\
\hline rel. error $D_{i o n}^{10 n}$ & $5.73 \%$ & $4.26 \%$ & $1.6 \%$ & $1.4 \%$ \\
\hline$t_{\exp } / \min$ & 119.3 & 63.2 & 1260.1 & 417.9 \\
\hline
\end{tabular}

frequency limits $\xi=\left[f_{1}, f_{P}\right]$, we are able to reduce the error in all estimated parameters by approximately $20 \%$ (Table 3 ). Coincidentally this results also in a reduction of about $20 \%$ of the experimental time. The optimum for this criterion is achieved for a frequency sweep that is well within the box constraints $f_{\min }$ and $f_{\mathrm{MAX}}$ as reported in Fig. 7.

It is also worthwhile giving a detailed analysis of the effect of the selection of the minimum and maximum frequency according to this optimization protocol: in Fig. 8 we vary the two extrema of the frequency spectrum and assume that the intermediate frequencies are spanned logarithmically with $P=50$ points. Fig. 8 shows a side by side relationship between data quality (the trace of $\mathbf{V}_{\text {asympt }}$ ) and experimental time, with the latter dominated by $f_{\min }$, the low-end frequency. It is easy to notice the presence of distinct minimum for the trace of $\mathbf{V}_{\text {asympt. }}$. This rather shallow minimum is related to a decrease in total error and it is mainly correlated with a decrease in the minimum frequency. The impedance for this case is presented in Fig. 7.

Below, we further relax constraints on the choice of frequencies. Specifically, we only require the frequencies to fall within the box constraint defined in (8). Furthermore, we allow frequency points to be repeated. In addition to the optimization condition we added a nonlinear constraint bounding the experimental time to be less than the time of the logarithmic distribution of frequencies $\mathbf{f}_{\log }$.
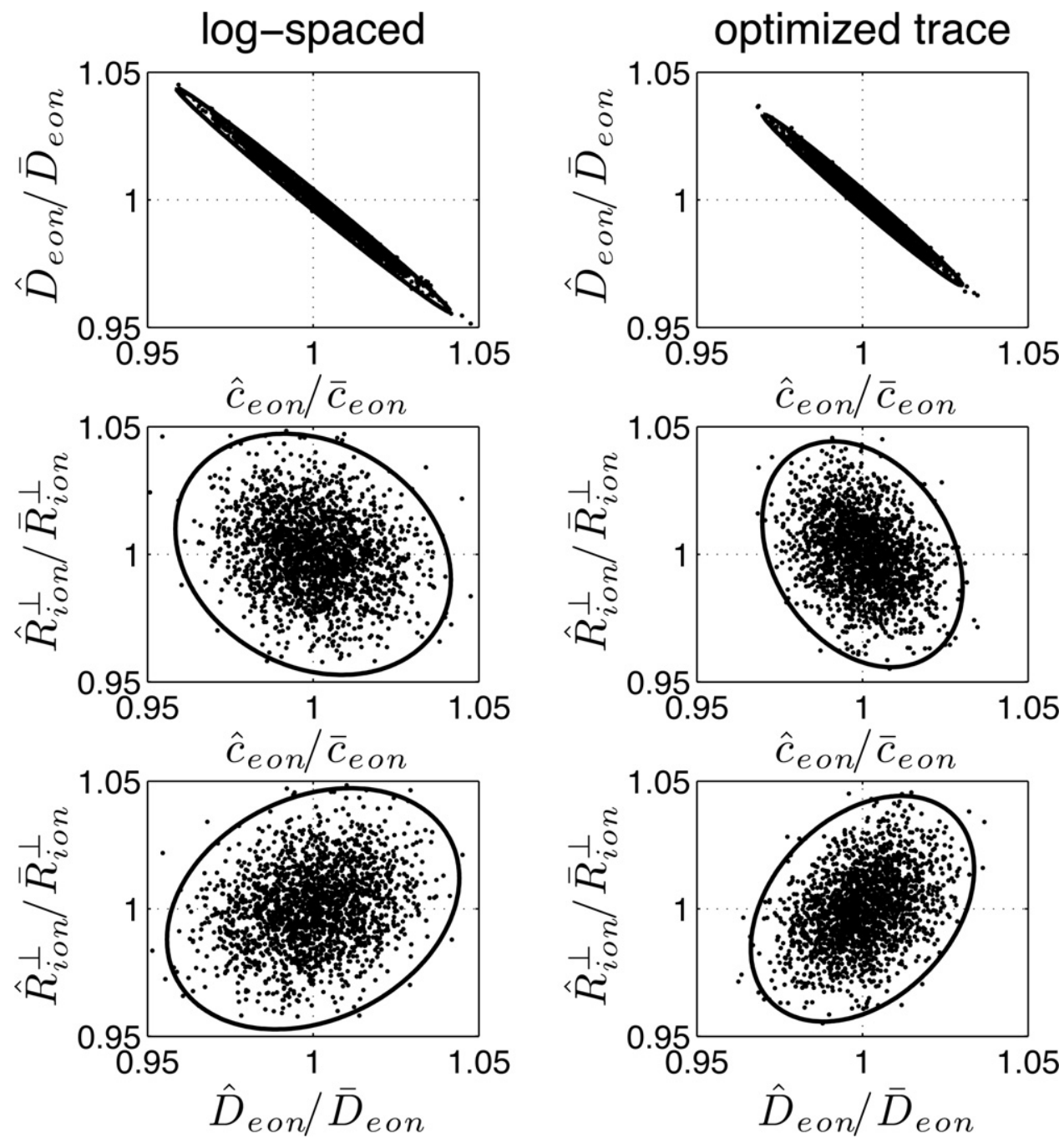

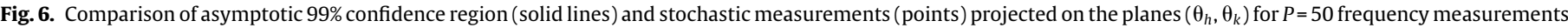

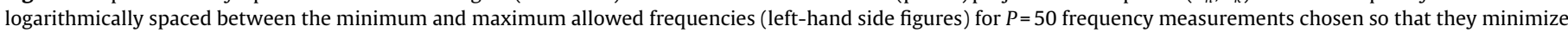

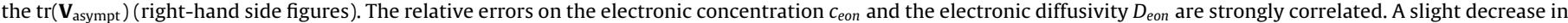
error is achieved by minimizing the trace. 

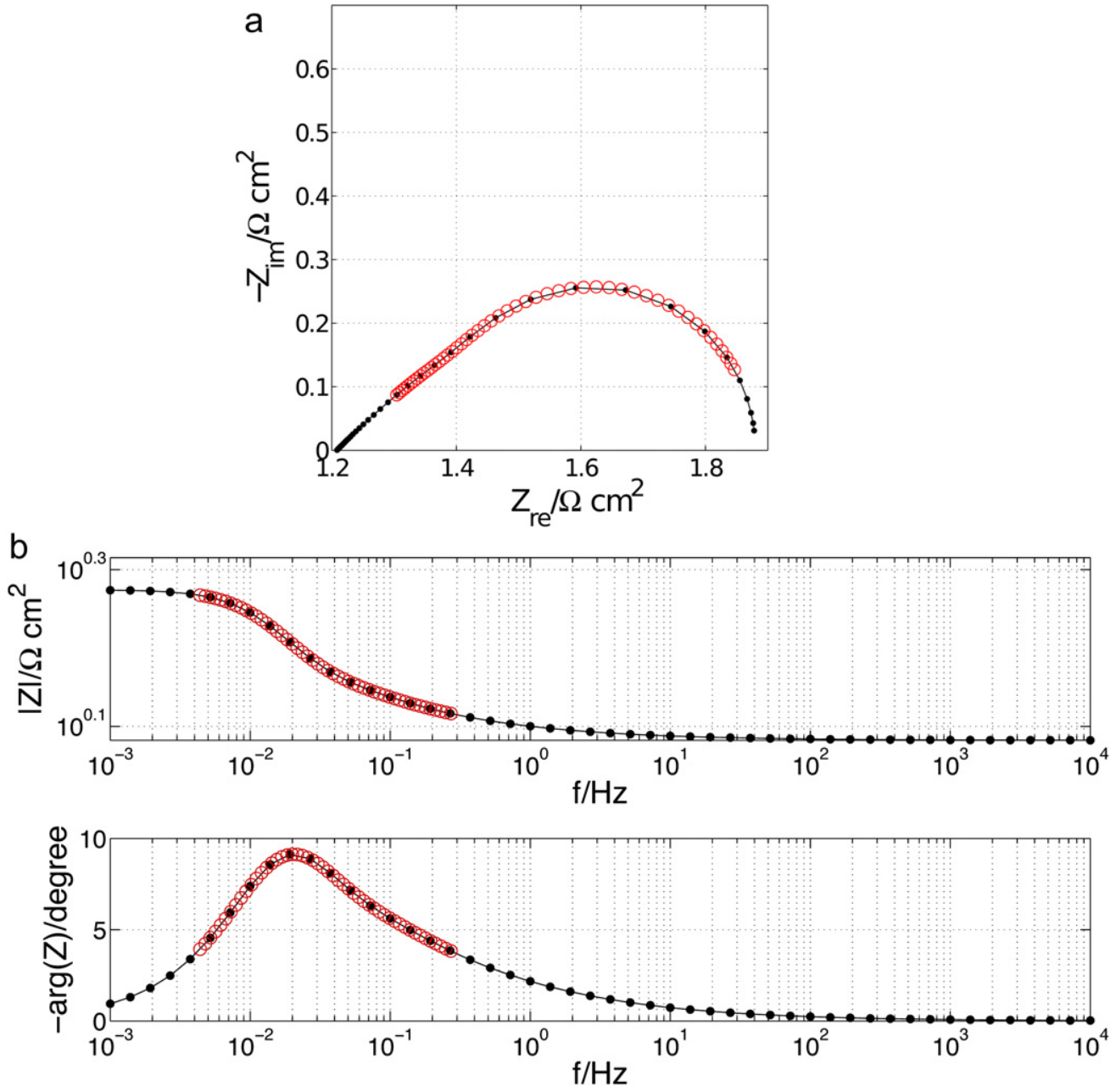

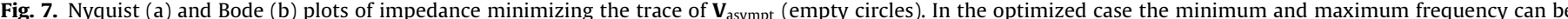

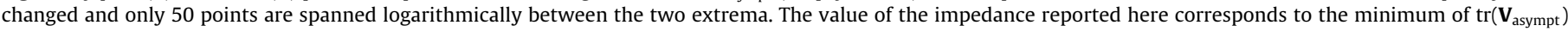
given in Fig. 8. The connected filled circled indicate the log-spaced impedance between minimum and maximum allowed frequency.
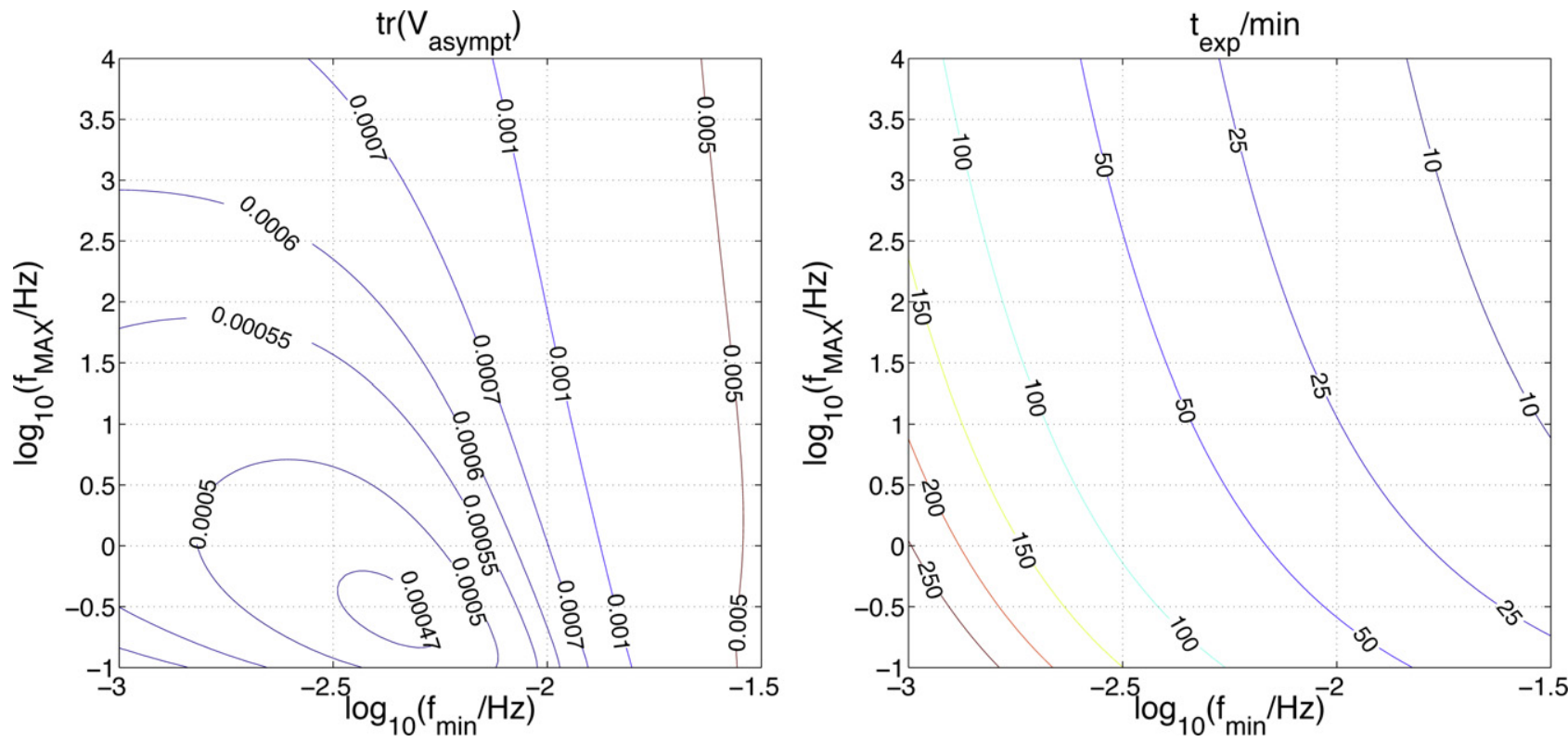

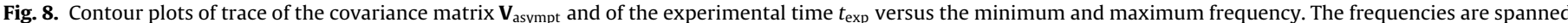

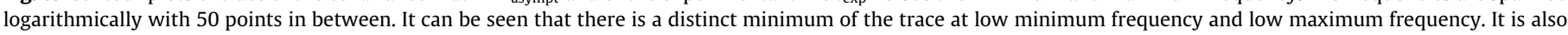
clear that the shorter the experimental time, the lower will be the quality of the data. 

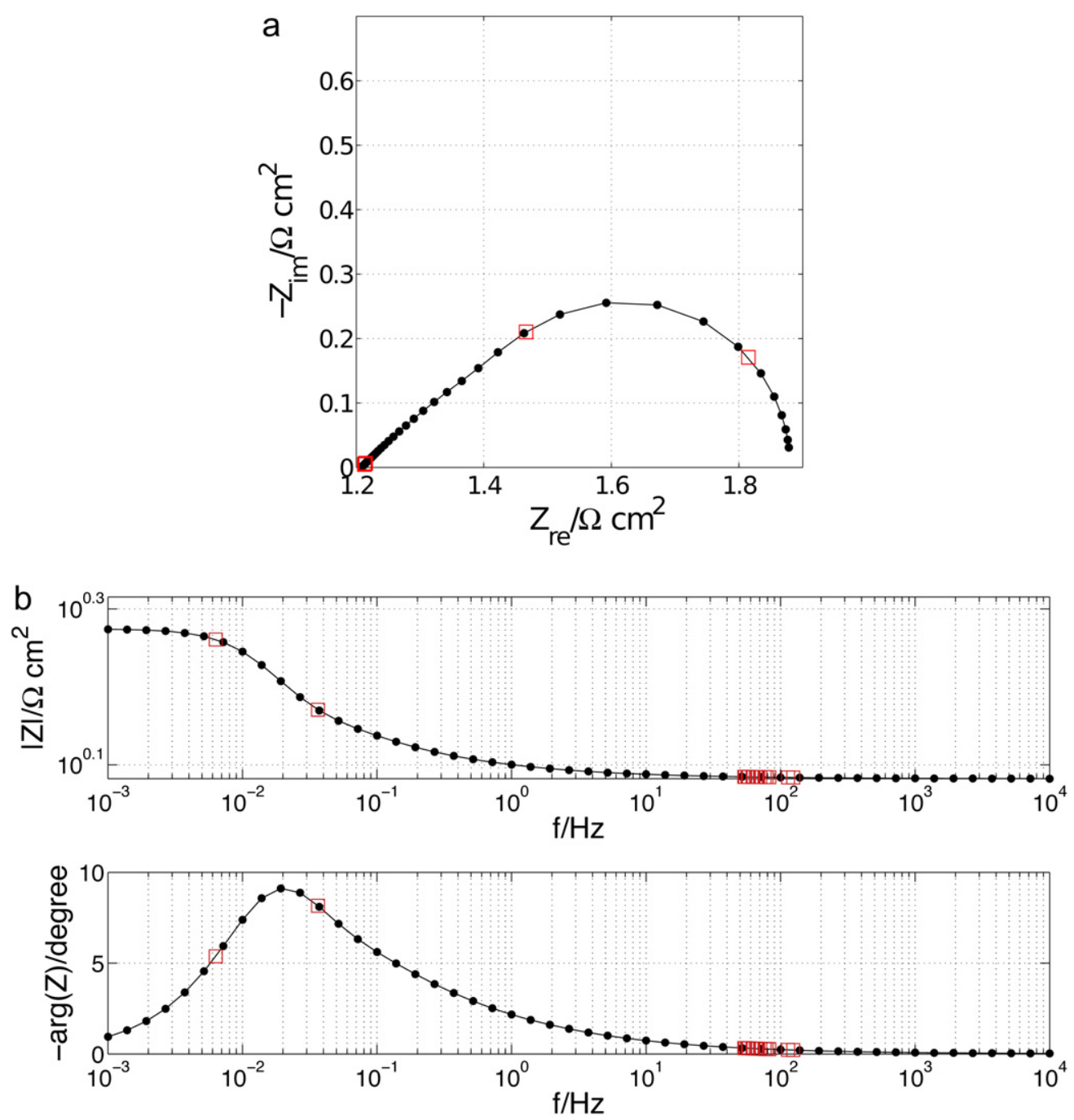

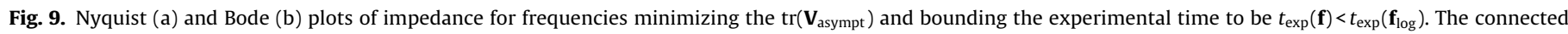
filled circled indicate the log-spaced impedance between minimum and maximum allowed frequency.

The result of this minimization is presented again in Table 3. It is apparent that by using OED not only the error decreases by approximately $30 \%$ with respect to the $\mathbf{f}_{\text {log }}$ case but also the time decreases by approximately $50 \%$ to $63.2 \mathrm{~min}$. The confidence region is also slightly reduced as shown in Fig. 6 .

More interestingly we can decrease the experimental time using the optimizer to select a set of frequencies that minimize experimental time. In other words we can search for the $\mathbf{f}=\arg \min _{\mathbf{f}} t_{\text {exp }}$ while bounding the trace as follows $\operatorname{tr}\left(\mathbf{V}_{\text {asympt }}\right) \leq \operatorname{tr}\left(\mathbf{V}_{\text {asympt }}\left(\mathbf{f}_{\text {log }}\right)\right)$, see Table 3. It is easy to see that, compared to the $\mathbf{f}_{\text {log }}$-case the time is reduced by more than a factor 4 (from 119.3 to $28.2 \mathrm{~min}$ ) and that the errors are approximately unvaried, see again Table 3.

It is helpful to have a look at the plots of the impedance in the Nyquist and Bode setting for the cases where the trace and the experimental time is minimized, Figs. 9 and 10 respectively. We notice that optimal EIS does not have a smoothly spaced set of frequencies and certain measurements are repeated. In Fig. 11, where the frequencies points are presented explicitly, it is apparent that the optimizer tends to give a non-smooth frequency spectrum where certain frequencies are lumped. It is also worthwhile noting that the upper and lower frequency ranges are approximately the same for both cases. Physically the lumping means that certain frequencies are more capable than others in reducing parameter errors. The observation that optimal frequency selection in EIS is substantially different than regular log spacing confirms the importance of OED. We envision the following strategy for carrying out OED: first run a regular logarithmic sweep and validate a model (the model can be either empirical or physics-based), then repeat the measurements at the most sensitive frequencies in order to improve the data quality. To the authors' knowledge this is the first work in the field that provides tools to determine the enhanced sensitivity region of the spectrum. Repeating measurements at those frequencies is a way of further improving our estimate of the parameters. Experimental time can also be reduced if OED is coupled with an ad hoc optimization strategy.

As stated above, better parameter confidence often correlates with longer time and vice versa. It is easy to note that in Fig. 11 the number of low frequency points is larger (and thus the experimental time greater) in the case where the trace is minimized than in the case where the time is minimized.

\subsection{OED and geometry}

In addition to optimizing $\mathbf{f}$, one could also optimize the sample geometry to reduce measurement errors, parameter correlation, and measurement time. In order to do that, one could aim to minimize the $\operatorname{tr}\left(\mathbf{V}_{\text {asympt }}\right)$ with respect to the fraction of exposed surface $r_{A}$ and to the thickness $L$. The nonlinear correlation between the couple $\left(r_{A}, L\right)$ and the trace is reported in Fig. 12. In this plot it 

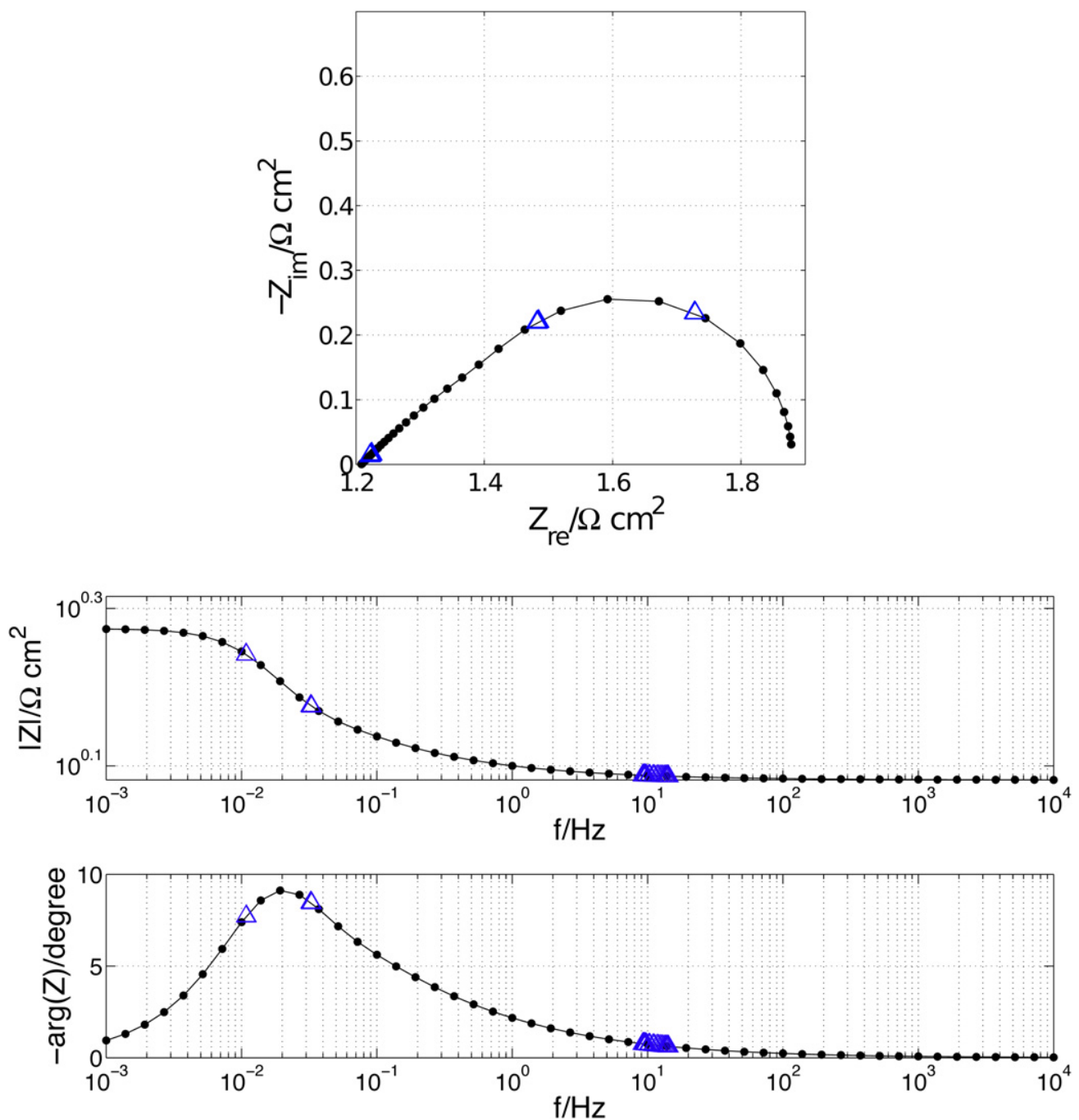

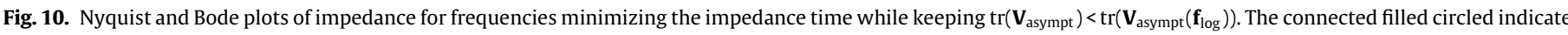
the log-spaced impedance between minimum and maximum allowed frequency.

is clearly shown a shallow minimum region centered at approximately $L=480 \mu \mathrm{m}$ and $r_{A}=0.8$. The trace is low for large thickness and exposed electrode area. The experimental data used in this work, $r_{A}=0.5$ and $L=770 \mu \mathrm{m}$, are robust with respect to parame-

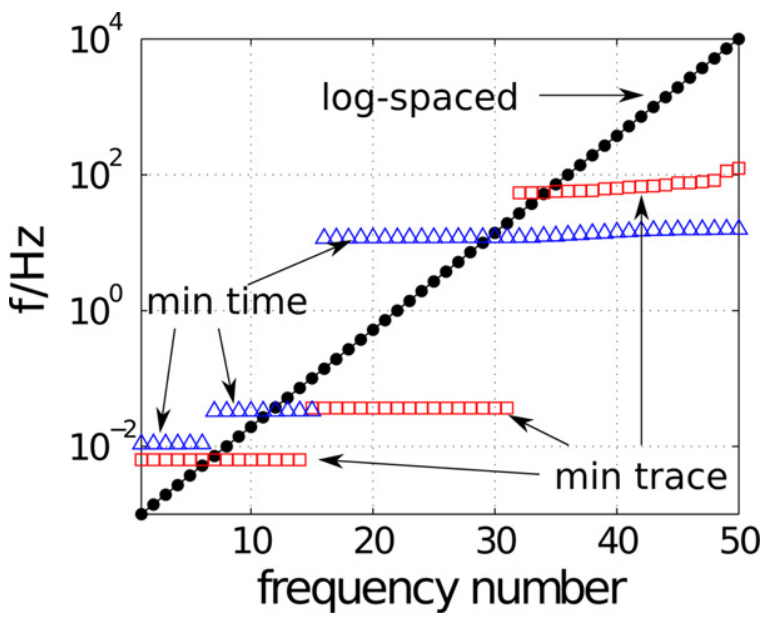

Fig. 11. Distribution of frequencies for the minimum trace (squares) and minimum time (triangles) cases assuming $P=50$ frequency points chosen. The solid line represents the log scale distribution of frequencies.

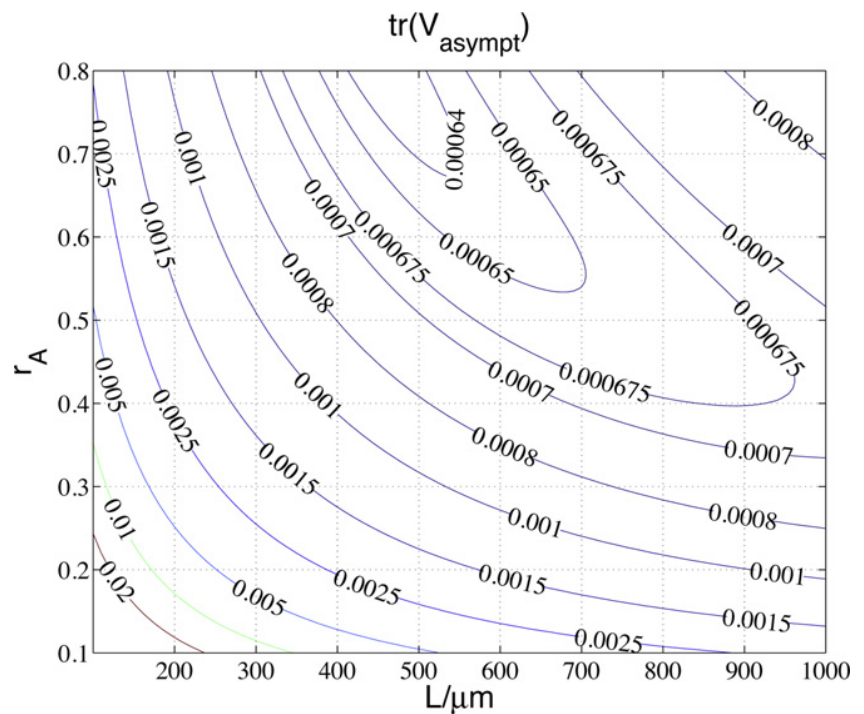

Fig. 12. Comparison of a priori designs of experiments; the frequencies are logarithmically distributed between the minimum and maximum bounds with 50 experimental points. 

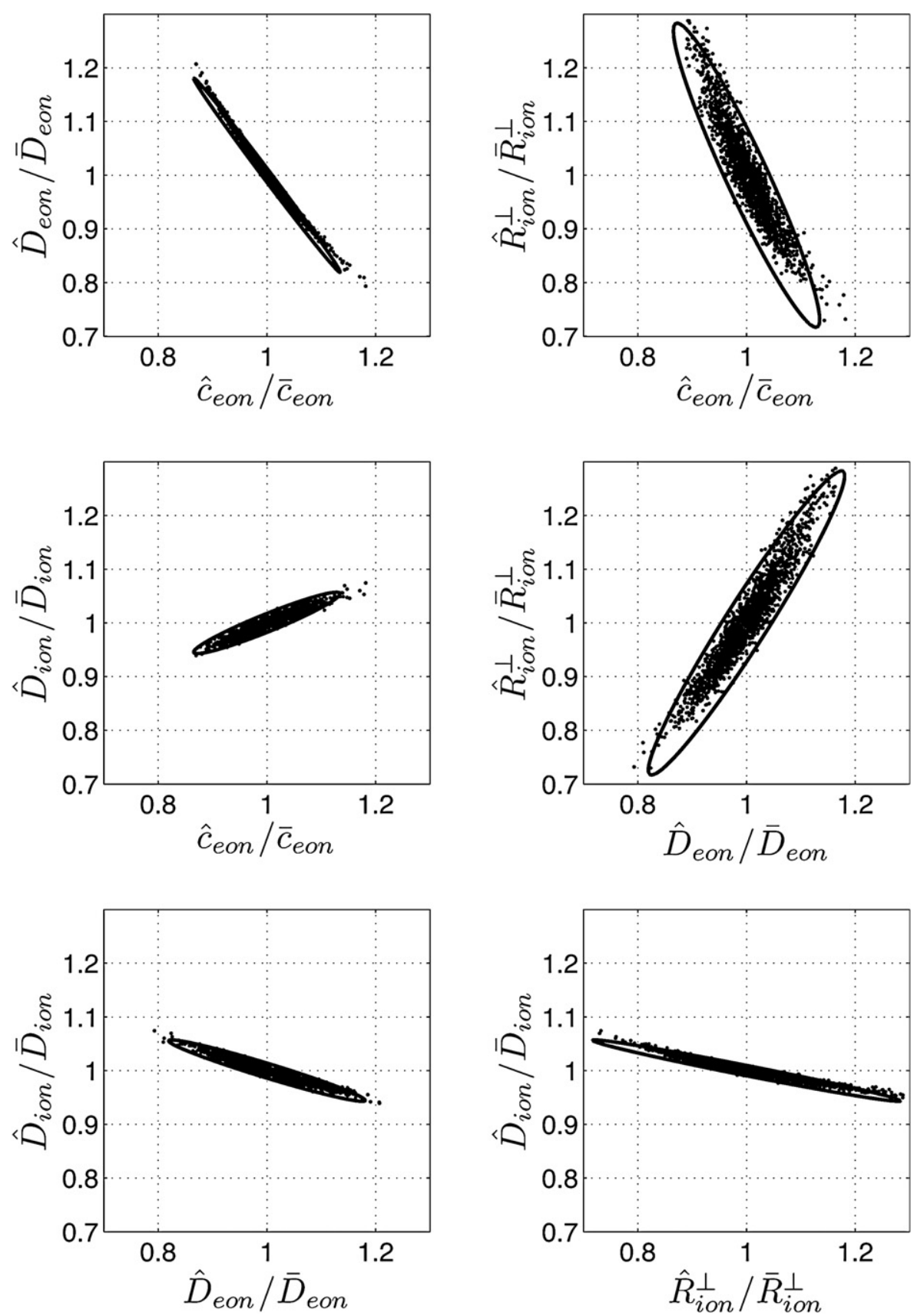

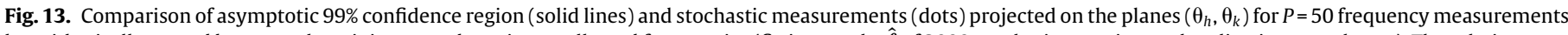

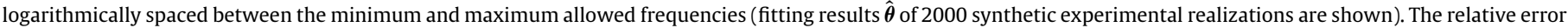
on the estimated parameters is quite large (at most 30\% at least 10\%). A large correlation among all parameters is shown and the $99 \%$ confidence ellipsoid is rather flat.

ter estimation. Samples of low thickness should be avoided as they are bound to have greater errors. Similarly small exposed area acts detrimentally with respect to parameter estimation.

\subsection{Estimation in the 4 parameter case}

As described earlier, in the experiments conducted by Lai and Haile [40] and Chueh et al. [42]. $D_{\text {ion }}$ was measured independently of the impedance presented earlier. The knowledge of $D_{\text {ion }}$ for this system can be achieved by a separate impedance measurement at sufficiently high $\mathrm{O}_{2}$ pressure and given dopant concentration. It is however worthwhile exploring the implications of estimating $D_{\text {ion }}$ from the given EIS experiment. By fitting also the $D_{\text {ion }}$ while keeping the 50 point logarithmic sweep $\mathbf{f}_{\text {log }}$ between minimum and maximum allowed frequencies, the errors on the parameters range from $10 \%$ to $30 \%$ as shown in the first column of Table 4 . They are considerably larger than the case where $D_{\text {ion }}$ is not included as a fitting parameter. The asymptotic confidence region and stochastically computed estimate distribution for this case are depicted in Fig. 13. The latter shows severe correlation between the parameters and an almost flat confidence ellipsoid as it can be seen by its projections. The asymptotic confidence region describes well stochastic confidence however slightly deviates from the ellipsoidal shape for large errors of parameter estimates.

In order to reduce the estimated parameter errors, we increased the number of sampling points from 50 to 600 and kept a logarithmic frequency distribution between $f_{\min }$ and $f_{\text {MAX }}$. This enhanced frequency span leads to a sharp decrease in error but it comes 

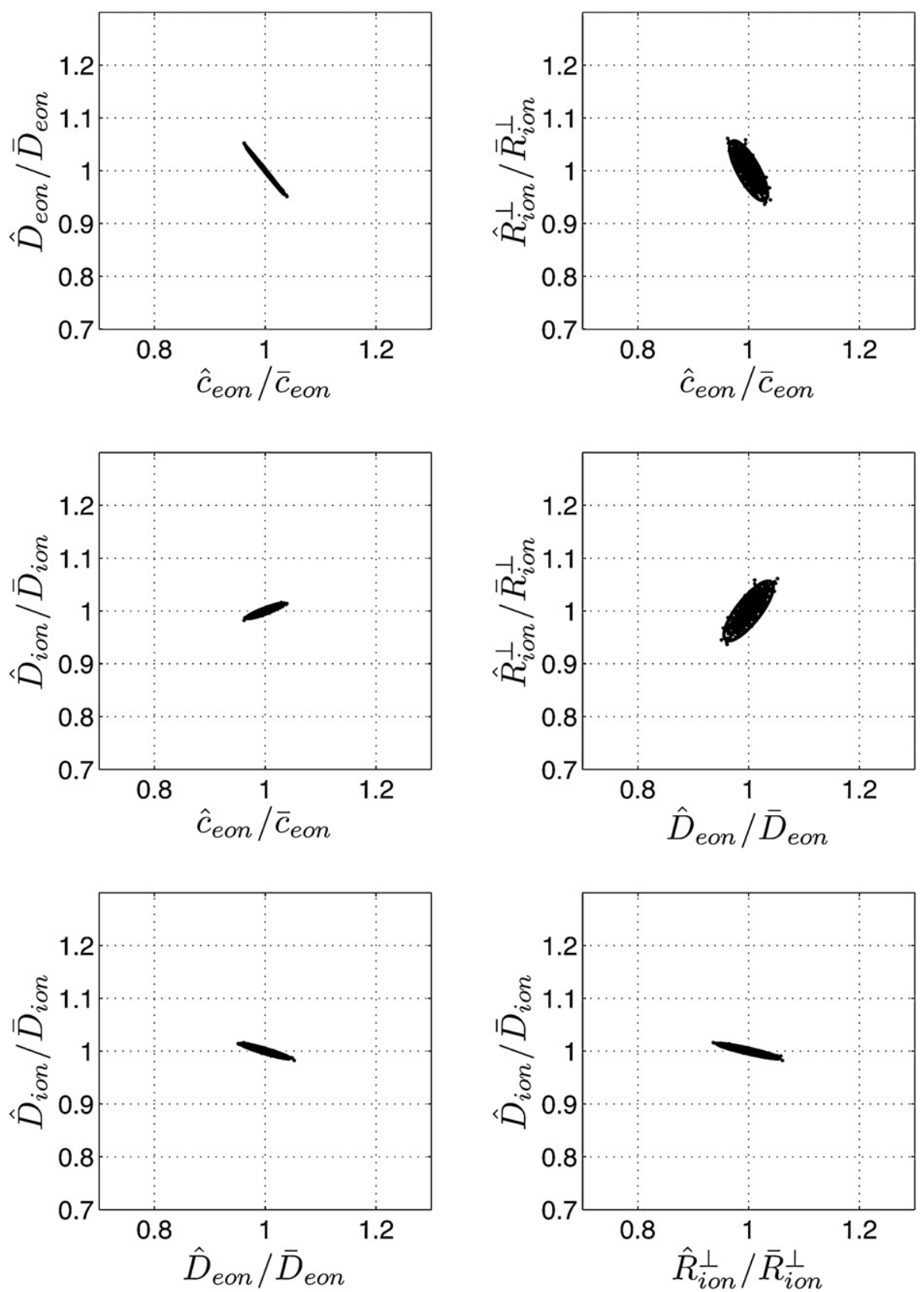

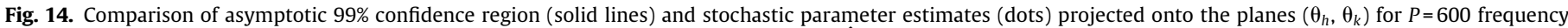

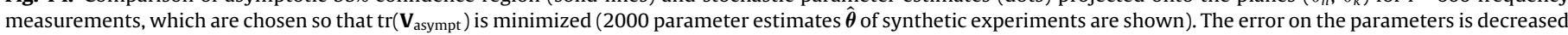
by an order of magnitude compared to Fig. 13. Minimizing the trace keeps a large correlation among some of the parameters.

at the cost of ten fold increase in time, see Table 4. By applying OED and minimizing the trace of the asymptotic covariance matrix with 600 frequency points that are free floating with a bound $t_{\exp }<5 \times t_{\log }$ we were able to reduce on one hand the errors on all parameters while keeping the experimental time to be less than four times the time of the initial $\mathbf{f}_{\log }$ experiment. This is also shown in Table 4 and in Fig. 14, the confidence region is reduced dramatically. However severe parameter correlations are present.

\subsection{Real time sequential design and experimental implementation}

Lastly, we wish to make a connection to a simulated experiment. The main idea behind the computational experiment is the ansatz that a decrease in variance can be achieved by placing the frequencies real time (or sequentially) just after each measurement is performed. The sequential approach is particularly suggested in situations (see Appendix B.2) where a priori knowledge of the parameters is not available or inadequate for OED purposes. The increased number of frequencies in the impedance sweep allows a better estimation of the parameters or, if the measured points are carefully selected, it leads to lowering the experimental time. The argument we use for the sequential optimization is that we probe the next frequency point based on OED at each measurement step. It is quite straightforward to note that the cost of high frequency measurements is quite low: according to the strategy employed in the experiment only if $f \leq 1 \mathrm{~Hz}$ then the time for capturing the impedance of one frequencies is below $2 \mathrm{~s}$. Specifically we ran the algorithm of Fig. 15. The computational experiment was repeated 2000 times at for each step, and the variances of experimental time and parameters estimates were computed. As it can 
Perform measurements at high frequency (low cost) choose $1 \mathrm{~Hz} \leq f_{p} \leq 10^{4} \mathrm{~Hz}$

$\log$ spaced with $p=1, \ldots, P=29$

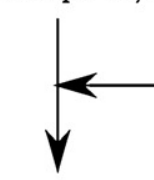

Estimate

$$
\hat{\boldsymbol{\theta}}_{P}=\arg \min _{\hat{\boldsymbol{\theta}}} \mathbb{S}_{P}
$$

where

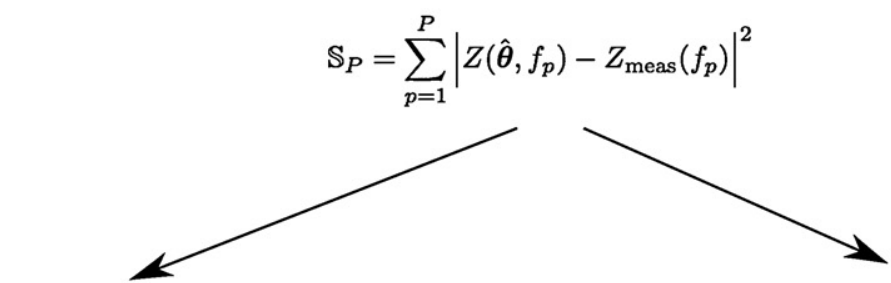

Determine (min time)

Determine (min trace)

$$
f_{P+1}=\arg \min _{f_{P+1}} t_{\exp }(\underbrace{f_{1}, \ldots, f_{P}}_{\text {fixed }}, f_{P+1})
$$

such that

$$
f_{P+1}=\arg \min _{f_{P+1}} \operatorname{tr}\left(V_{\text {asympt }}(\underbrace{\hat{\boldsymbol{\theta}}_{P}, f_{1}, \ldots, f_{P}}_{\text {fixed }}, f_{P+1})\right)
$$

such that

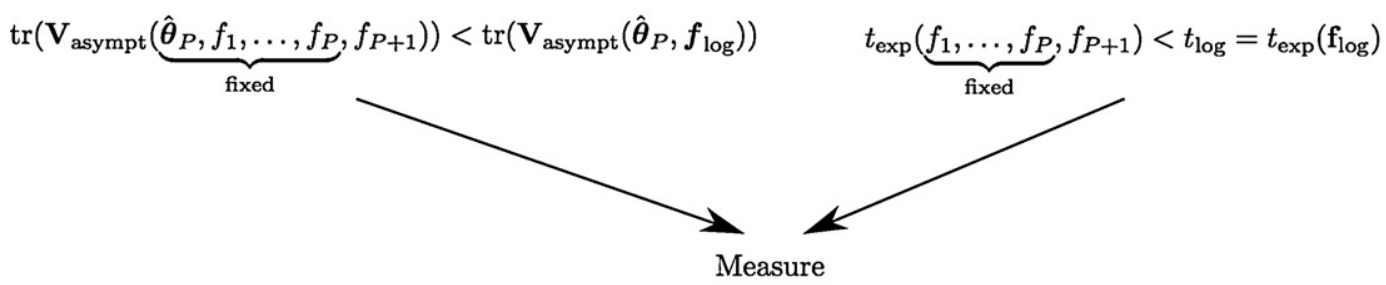

$$
Z_{\text {meas }}\left(f_{P+1}\right)
$$

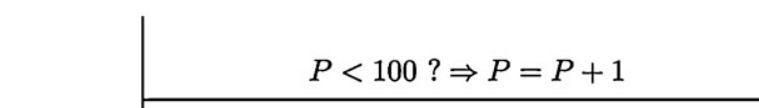

$P=100 ?$

Estimate

$$
\hat{\boldsymbol{\theta}}_{100}=\arg \min _{\hat{\boldsymbol{\theta}}} \mathbb{S}_{100}
$$

Fig. 15. Flow diagram for the sequential algorithm.

be seen in the last two columns Table 3, the error on the parameters and the time can be reduced significantly compared to the $\mathbf{f}_{\text {log }}$ frequency span (errors go from $4-5 \%$ to $2-4 \%$ and time goes from 119.3 to $36 \mathrm{~min}$ ). In Fig. 16 we provide the mean error of the parameter estimate as the experiments evolves (the time-reducing algorithm is shown). As the algorithm proceeds the errors decrease monotonically and the experimental time increases monotonically (we note a slight slope also for high frequency measurement $P$ ). It is shown that on average 60 frequency points are sufficient to achieve the same level of accuracy of the initial $\mathbf{f}_{\text {log }}$ experiment at approximately $1 / 3$ of the experimental time. The error decrease is sharpest for the $D_{\text {eon }}$, which is captured with large errors at the initial steps. The variance on the experimental time increases as the experiment proceeds and it is very likely that the experiment time ranges between 27 and $44 \mathrm{~min}$. We found as above that certain frequencies are more informative than others, in the sequential case we find that two peaks, one at $60 \mathrm{mHz}$ and the other one at $2.3 \mathrm{~Hz}$, tend on average to be repeated multiple time in sequential impedance experiments for time reduction, see Fig. 17. This last result is in partial agreement with what is reported in Fig. 11 and it is indicative of the presence of many local minima in the constrained time minimization case. The local optimizer can only reach a subset of those and it is experiment-dependent (the actual values of $\boldsymbol{\theta}$ used in the two are different, in one case it is the exact value, in the other case, it is the estimated value $\hat{\boldsymbol{\theta}}$ ). 

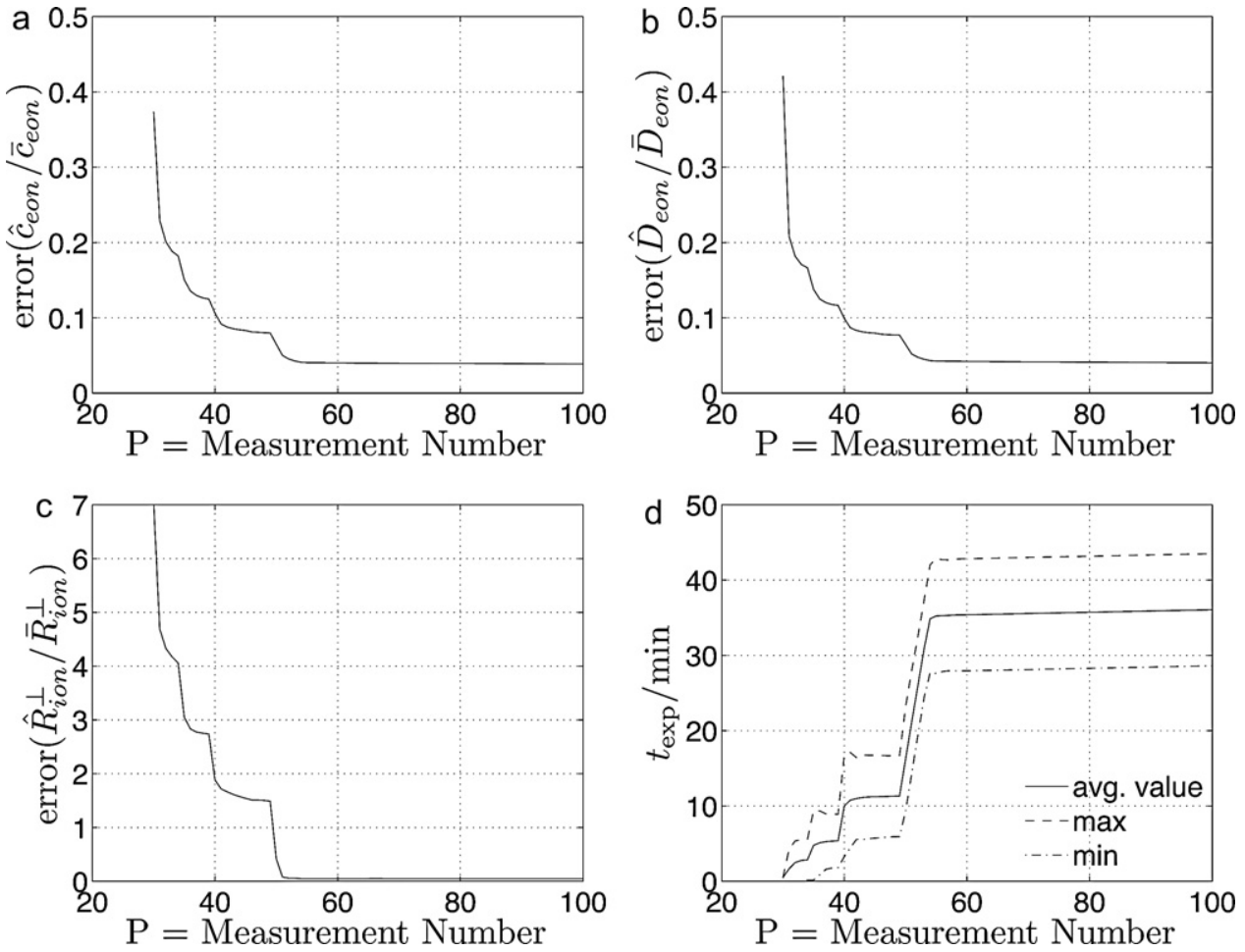

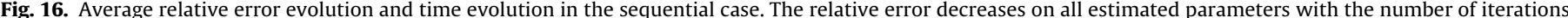

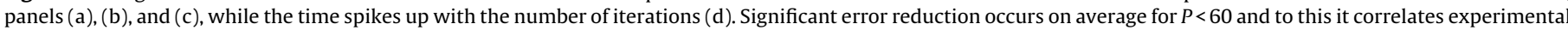
time increase.

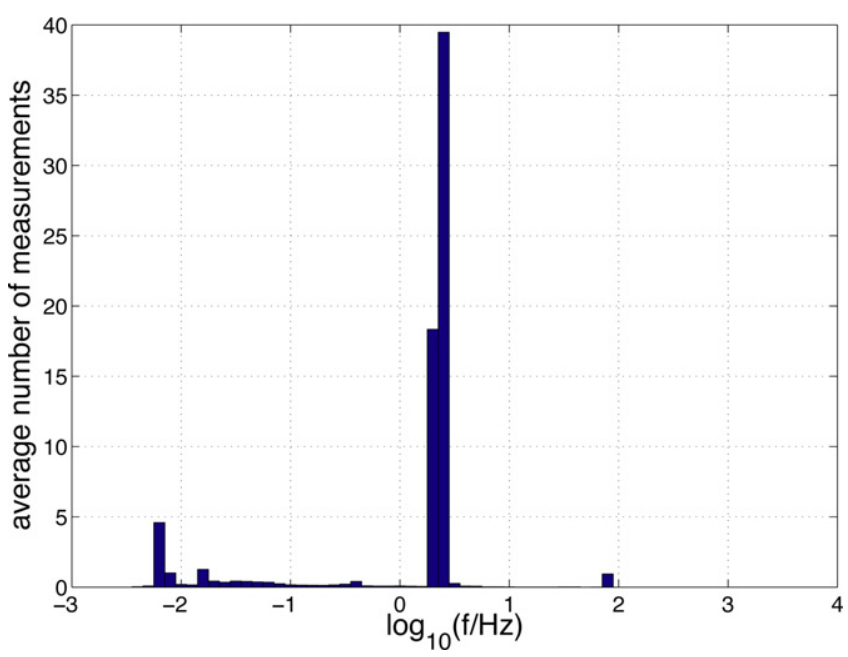

Fig. 17. Number of times a certain frequency is repeated on average in each experiment, each measurement is binned into intervals that span from $f_{\min }$ to $f_{\max }$ and have 10 intervals per decade. Frequencies below $P<60$ are not considered. Two major peaks emerge, where frequencies are repeated during an experiment, one at about $2.5 \mathrm{~Hz}$ and the centered around $0.63 \mathrm{~Hz}$.

\section{Conclusions}

In this paper we have shown several novel methods for the reduction of inaccuracies in physical parameters estimated from electrochemical impedance spectroscopy. We developed an asymptotic covariance estimator $\mathbf{V}_{\text {asympt }}$, which in the case of small errors, describes well the confidence region of estimated parameters $\hat{\boldsymbol{\theta}}$. We used this approach to capture the error propagation from the measurement error space (the $\sigma$ 's) to the estimated parameter space $(\hat{\boldsymbol{\theta}})$. In the 4 parameter estimation case we have shown that small measurement errors $(0.5 \%$ in the amplitude and $3 \%$ in the phase) can lead to significant inaccuracies in the estimated parameters. By minimizing the trace of the asymptotic covariance matrix $\left(\mathbf{V}_{\text {asympt }}\right)$ errors in estimated parameters were reduced significantly. In certain cases, the experimental time were also reduced. We also simulated experiments and produced a sequential algorithm, which, with given information about the experiment model, Eq. (2), and experimental error, is able to reduce the errors of the estimated parameters "on the fly".

The current methodology can be further enhanced by weighing the terms summed in the least squares functional and by optimizing this weighing process. While non-uniform weights are frequently used, OED can provide methods to decrease the confidence region size even further by tweaking simultaneously those with frequently placement. The error structure asymptotic estimate can be further improved by applying a Bayesian approach [17]. Lastly model discrimination can be used in a sequential manner to improve initial parametrical estimates [64].

We believe that the tools developed in this manuscript can significantly help enhance experiments in the following way:

1. One can aim at understanding the correlation between EIS experimental errors and estimated parameter errors. Specifically the experimentalist can analyze the measured data to find parameter errors and correlations by means of the expression (C.15) for $\mathbf{V}_{\text {asympt. }}$.

2. The experimentalist can aim at decreasing errors in the estimated parameters. One can determine the statically more sensitive frequencies for the experiments, and if needed, repeat the experiment at those frequencies to increase the confidence on the estimated parameters

3. The experimentalist could speed up EIS experiments. This can be achieved by coupling in real-time the estimation process and the measurements. For this purpose the sequential algorithm provided in Fig. 15 can be used. This approach would lead 
to increased accuracy in parameter estimation and significant reduction in experimental time.

\section{Acknowledgements}

F.C. acknowledges that the Marie Curie Reintegration Grant FastCell-256583 partially supported his work. T.C. was supported by Deutsche Forschungsgemeinschaft (DFG) through the project Modellierung, Simulation und Optimierung der Mikrostruktur mischleitender SOFC-Kathoden. W.C.C. was supported in part by an appointment to the Sandia National Laboratories Truman Fellowship in National Security Science and Engineering, sponsored by Sandia Corporation (a wholly owned subsidiary of Lockheed Martin Corporation) as Operator of Sandia National Laboratories under its U.S. Department of Energy Contract No. DE-AC04-94AL85000. W.L. thanks Michigan State University for providing the start-up package.

\section{Appendix A. Nomenclature}

$\begin{array}{ll}c_{e o n} & \text { electron concentration } \\ c_{i o n} & \text { vacancy concentration } \\ \mathbf{f} & \text { vector of frequencies used in an impedance measurement } \\ k_{B} & \text { Boltzmann constant } \\ L & \text { sample thickness } \\ r_{A} & \text { fraction of electrocatalytic area exposed to the gas } \\ R_{i o n}^{\perp} & \text { area specific resistance } \\ t_{\text {exp }} & \text { time necessary to perform the impedance experiment } \\ t_{\text {log }} & \text { time necessary to perform the impedance experiment } \\ \operatorname{tr}(\mathbf{A}) & \text { with log-spaced frequencies } \\ T & \text { trace of the matrix } \mathbf{A} \\ \boldsymbol{V} & \text { temperature } \\ \boldsymbol{V}_{\text {asympt }} & \text { asymptotic covariance matrix } \\ \boldsymbol{\theta} & \text { vector of the model parameters } \\ \overline{\boldsymbol{\theta}} & \text { vector of the experimental parameters } \\ \hat{\boldsymbol{\theta}} & \text { vector of the estimated parameters } \\ \boldsymbol{\theta}_{\mathrm{dim}} & \text { vector of the model parameters (dimensional) } \\ \kappa(\mathbf{A}) & \text { condition number of the matrix } \mathbf{A} \text { defined as }\left|\frac{\text { max } \lambda(A)}{\min \lambda(A)}\right| \\ \lambda(\mathbf{A}) & \text { list of eigenvalues of the matrix } \mathbf{A} \\ \boldsymbol{\xi} & \text { vector of the design parameters } \\ \sigma_{A} & \text { relative error(standard deviation) on the amplitude of the } \\ \sigma_{\phi} & \text { measured impedance } \\ \boldsymbol{\Xi} & \text { relative error (standard deviation) on the phase angle of } \\ & \text { the measured impedance } \\ & \text { design space }\end{array}$

\section{Appendix B. Brief review of OED}

\section{B.1. The linear case}

Let's consider the multivariate linear case, i.e., a model of the type $\mathbf{Y}_{\text {model }}(\boldsymbol{\theta})=\mathbf{Q} \boldsymbol{\theta}$, with $\mathbf{Y}_{\text {model }} \in \mathbb{R}^{N}$ the observable quantity, $\boldsymbol{\theta} \in \mathbb{R}^{N_{\theta}}$ the vector of model parameters and $\mathbf{Q} \in \mathbb{R}^{N \times N_{\theta}}$ the matrix that links the parameters to the measurement. To simplify the exposition we consider the simple model error of the type

$\mathbf{Y}_{\text {meas }}(\boldsymbol{\theta})=\mathbf{Y}_{\text {model }}(\boldsymbol{\theta})+\boldsymbol{\epsilon}$,

where each $\mathbb{R}^{N}$-valued measurement error $\boldsymbol{\epsilon}$ is normally distributed $\mathcal{N}\left(0, \Sigma_{\epsilon}\right)$, with zero mean and covariance matrix $\boldsymbol{\Sigma}_{\epsilon}$. In the linear case normally distributed errors $\boldsymbol{\epsilon}$ lead to normal estimated parameters $\hat{\boldsymbol{\theta}}$ [34]. In this case the least-squares solution gives an unbiased estimation of the parameters $\hat{\boldsymbol{\theta}}$, with posterior covariance given by

$\mathbf{V}=\left(\mathbf{Q}^{\mathrm{T}} \boldsymbol{\Sigma}_{\epsilon}^{-1} \mathbf{Q}\right)^{-1}$

The OED method aims at minimizing a function of $\mathbf{v}$ by the determining and implementing the most informative experimental conditions.

It is important to note that $\mathbf{V}$ does not depend on $\boldsymbol{\theta}$, so an OED problem can be solved for an estimated value $\hat{\boldsymbol{\theta}}$ of the parameters.

\section{B.2. The nonlinear case}

In the nonlinear case the model is

$\mathbf{Y}_{\text {model }}(\boldsymbol{\theta})=\boldsymbol{\eta}(\boldsymbol{\theta})$,

with a nonlinear function $\boldsymbol{\eta}(\boldsymbol{\theta})$, while the Jacobian of the observation (the matrix of the derivatives of $\boldsymbol{Y}(\boldsymbol{\theta})$ with respect to the parameters) is

$\mathbf{Q}_{i j}=\frac{\partial \boldsymbol{\eta}_{i}(\boldsymbol{\theta})}{\partial \theta_{j}}$.

There are at least two issues to be kept in mind for the general nonlinear case

- an expression of the covariance matrix similar to (B.2) is only valid in an asymptotic sense;

- the Jacobian $\boldsymbol{Q}$ is a function of $\boldsymbol{\theta}$, a value typically not available to the experimenter. The value often known is instead $\hat{\boldsymbol{\theta}}$ a natural strategy to solve an OED problem is to proceed sequentially. One applies OED to improve estimates of the exact parameters $\theta$ [65-67].

\section{B.3. Approximation of the confidence region}

The confidence region gives a measure of the statistical goodness of the estimator. The least squares function $\mathbb{S}(\boldsymbol{\theta})$ has its minimum at $\hat{\boldsymbol{\theta}}$, which is the solution of the least squares problem for a given set of measurements. The so-called confidence region describes the region around the estimated parameter $\hat{\boldsymbol{\theta}}$ in which it is likely to find the exact parameter.

The confidence region with level $K$ can be defined as the set of parameters that satisfies the following relation:

$\Theta=\left\{\boldsymbol{\theta}^{\prime}: \mathbb{S}\left(\boldsymbol{\theta}^{\prime}\right)-\mathbb{S}(\hat{\boldsymbol{\theta}}) \leq K\right\}$,

for a statistical definition of $K$ see a classical textbook [61,68]. In the general nonlinear case the region $\Theta$ defined in the last equation may have not a simple shape and may not be a connected set. However, if the error in the data is small enough (in a typical experiment this can to be checked only a posteriori), one can use the linearized confidence region. If one defines $\boldsymbol{\theta}^{\prime}=\delta \boldsymbol{\theta}+\hat{\boldsymbol{\theta}}$ and takes the second order expansion of the expression, one will obtain that

$$
\begin{aligned}
\mathbb{S}\left(\boldsymbol{\theta}^{\prime}\right)-\mathbb{S}(\hat{\boldsymbol{\theta}}) & =\frac{\partial \mathbb{S}(\hat{\boldsymbol{\theta}})}{\partial \hat{\boldsymbol{\theta}}} \delta \boldsymbol{\theta}+\delta \boldsymbol{\theta}^{T} \frac{\partial^{2} \mathbb{S}(\hat{\boldsymbol{\theta}})}{\partial \hat{\boldsymbol{\theta}}^{2}} \delta \boldsymbol{\theta}+O\left(\|\delta \boldsymbol{\theta}\|^{2}\right) \\
& =+\delta \boldsymbol{\theta}^{T} \frac{\partial^{2} \mathbb{S}(\hat{\theta})}{\partial \boldsymbol{\theta}^{2}} \delta \boldsymbol{\theta}+O\left(\|\delta \boldsymbol{\theta}\|^{2}\right)
\end{aligned}
$$

since $\partial \mathbb{S}(\hat{\boldsymbol{\theta}}) / \partial \boldsymbol{\theta}=0$. This gives that

$\Theta_{\text {Lin }}=\left\{\hat{\boldsymbol{\theta}}+\delta \boldsymbol{\theta}: \delta \boldsymbol{\theta}^{T} \frac{\partial^{2} \mathbb{S}(\hat{\boldsymbol{\theta}})}{\partial \boldsymbol{\theta}^{2}} \delta \boldsymbol{\theta} \leq K\right\}$,

where $\partial^{2} \mathbb{S}(\hat{\boldsymbol{\theta}}) / \partial \boldsymbol{\theta}^{2}$ is the matrix of second derivatives (the Hessian). 
In the linear case the expression (B.2) corresponds to the inverse of the Hessian of the least squares function [31]. In case of Independently distributed errors the expression

$\Theta_{\text {Lin }}=\left\{\hat{\boldsymbol{\theta}}+\delta \boldsymbol{\theta}: \delta \boldsymbol{\theta}^{T} \mathbf{V}_{\text {asympt }}^{-1} \delta \boldsymbol{\theta} \leq K\right\}$.

can be used to represent the confidence region in the parameter space.

In a nonlinear model the estimated parameters are not necessarily normally distributed even in the case of additive and normal errors. If the nonlinearity in the model is weak, then the latter can be linearized and the posterior probability density of the estimated parameters is asymptotically Gaussian [69-71]. This occurs for example if the measurement errors are sufficiently small. In this case one can use expressions analogous to (B.2) in order to calculate the linearized confidence region [30,31]. The latter has an interesting geometrical interpretation: since $\mathbf{V}$ is a positive definite matrix in $\mathbb{R}^{N_{\theta} \times N_{\theta}}$, its representation in $\boldsymbol{\theta}$ space is an ellipsoid centered at the estimated values of the parameters $\hat{\boldsymbol{\theta}}$ with semiaxis given by the eigenvalues of $\mathbf{V}$.

\section{B.4. Minimization criteria}

The typical criteria for determining the function to be minimized $\Phi$ given in (1) are [17]

1. A-criterion (trace): $\Phi=\operatorname{tr}\left(\mathbf{V}_{\text {asympt }}\right)$, which corresponds to the summation of the semiaxis of the confidence region (a reduction of $\operatorname{tr}\left(\mathbf{V}_{\text {asympt }}\right)$ corresponds to the decrease of the sum of the variances of all parameters);

2. D-criterion (determinant): $\Phi=\operatorname{det}\left(\mathbf{V}_{\text {asympt }}\right)$, which corresponds to the minimization of the volume of the confidence region;

3. E-criterion (maximum eigenvalue): $\Phi=\max \lambda\left(\mathbf{V}_{\text {asympt }}\right)$, which is the minimization of the biggest semiaxis of the confidence region;

4. Modified E-criterion: $\Phi=\kappa\left(\mathbf{V}_{\text {asympt }}\right)$, where $\kappa$ is the condition number of $\mathbf{V}$. This is especially used in biological applications and in large systems where one aims at lowering parameter correlations [32].

\section{Appendix C. Derivation of the asymptotic covariance matrix}

Define the model experiment output

$Z_{\text {meas }}=Z(\overline{\boldsymbol{\theta}}, f, \boldsymbol{\epsilon})$,

where $\epsilon$ is $\mathbb{R}^{2}$ valued and normally distributed $\left(\boldsymbol{\epsilon} \sim \mathcal{N}\left(0, \boldsymbol{\Sigma}_{\epsilon}\right)\right), \overline{\boldsymbol{\theta}}$ is the (column) vector of "true" parameters, which are "unknown" to the experimenter and $f$ is the tunable parameter (we have omitted all other known input parameters). The model used for fitting the data is given by

$Z(\boldsymbol{\theta}, f, \boldsymbol{\epsilon}=0)$

The goal of the estimation is to find the parameter vector $\hat{\boldsymbol{\theta}}^{5}$ that is close to the "true" $\overline{\boldsymbol{\theta}}$. This is achieved by seeking the minimum of the distance between the experimental data and the model given by the following sum of squares $\mathbb{S}$ :

$$
\begin{aligned}
\mathbb{S}(\boldsymbol{\theta}, \overline{\boldsymbol{\theta}}) & =\sum_{p=1}^{P}\left|Z\left(\boldsymbol{\theta}, f_{p}, \boldsymbol{\epsilon}=0\right)-Z\left(\overline{\boldsymbol{\theta}}, f_{p}, \boldsymbol{\epsilon}_{p}\right)\right|^{2} \\
& =\sum_{\substack{P=1\\
}}\left(Z\left(\boldsymbol{\theta}, f_{p}, \boldsymbol{\epsilon}=0\right)-Z\left(\overline{\boldsymbol{\theta}}, f_{p}, \boldsymbol{\epsilon}_{p}\right)\right)^{*}\left(Z\left(\boldsymbol{\theta}, f_{p}, \boldsymbol{\epsilon}=0\right)\right. \\
& \left.-Z\left(\overline{\boldsymbol{\theta}}, f_{p}, \boldsymbol{\epsilon}_{p}\right)\right) .
\end{aligned}
$$

\footnotetext{
${ }^{5}$ It is important to note that $\hat{\boldsymbol{\theta}}$ is a random variable.
}

The minimizing $\hat{\boldsymbol{\theta}}$ is then defined as

$\hat{\boldsymbol{\theta}}=\underset{\boldsymbol{\theta}}{\operatorname{argmin}} \mathbb{S}(\boldsymbol{\theta}, \overline{\boldsymbol{\theta}})$,

which in turn satisfies

$$
\left.\frac{\partial \mathbb{S}(\boldsymbol{\theta}, \overline{\boldsymbol{\theta}})}{\partial \boldsymbol{\theta}}\right|_{\boldsymbol{\theta}=\hat{\boldsymbol{\theta}}}=0 .
$$

If we use the latter expression in (C.3) we shall get that

$$
\begin{aligned}
0= & \left.\frac{\partial \mathbb{S}(\boldsymbol{\theta}, \overline{\boldsymbol{\theta}})}{\partial \boldsymbol{\theta}}\right|_{\boldsymbol{\theta}=\hat{\boldsymbol{\theta}}}=\sum_{p=1}^{P}\left(\left.\frac{\partial Z\left(\boldsymbol{\theta}, f_{p}, \boldsymbol{\epsilon}=0\right)}{\partial \boldsymbol{\theta}}\right|_{\boldsymbol{\theta}=\hat{\boldsymbol{\theta}}}\right)^{*}\left(Z\left(\hat{\boldsymbol{\theta}}, f_{p}, \boldsymbol{\epsilon}=0\right)\right. \\
& \left.-Z\left(\overline{\boldsymbol{\theta}}, f_{p}, \boldsymbol{\epsilon}_{p}\right)\right)+\left.\sum_{p=1}^{P}\left(Z\left(\hat{\boldsymbol{\theta}}, f_{p}, \boldsymbol{\epsilon}=0\right)-Z\left(\overline{\boldsymbol{\theta}}, f_{p}, \boldsymbol{\epsilon}_{p}\right)\right)^{*} \frac{\partial Z\left(\boldsymbol{\theta}, f_{p}, \boldsymbol{\epsilon}=0\right)}{\partial \boldsymbol{\theta}}\right|_{\boldsymbol{\theta}=\hat{\boldsymbol{\theta}}}
\end{aligned}
$$

We now apply the asymptotic hypothesis and suppose that the error is "small", i.e., we define $\delta \boldsymbol{\theta}=\hat{\boldsymbol{\theta}}-\overline{\boldsymbol{\theta}}$ and we suppose that the following three hypotheses hold:

$$
\begin{aligned}
Z\left(\hat{\boldsymbol{\theta}}, f_{p}, \boldsymbol{\epsilon}=0\right) \approx Z\left(\overline{\boldsymbol{\theta}}, f_{p}, \boldsymbol{\epsilon}=0\right)+\left.\frac{\partial Z\left(\boldsymbol{\theta}, f_{p}, \boldsymbol{\epsilon}=0\right)^{T}}{\partial \boldsymbol{\theta}}\right|_{\boldsymbol{\theta}=\overline{\boldsymbol{\theta}}} \delta \boldsymbol{\theta}, \\
Z\left(\overline{\boldsymbol{\theta}}, f_{p}, \boldsymbol{\epsilon}_{p}\right) \approx Z\left(\overline{\boldsymbol{\theta}}, f_{p}, \boldsymbol{\epsilon}=0\right)+\frac{\partial Z\left(\overline{\boldsymbol{\theta}}, f_{p}, \boldsymbol{\epsilon}=0\right)^{T}}{\partial \boldsymbol{\epsilon}} \boldsymbol{\epsilon}_{p}, \\
\left.\left.\frac{\partial Z\left(\boldsymbol{\theta}, f_{p}, \boldsymbol{\epsilon}=0\right)}{\partial \boldsymbol{\theta}}\right|_{\boldsymbol{\theta}=\hat{\boldsymbol{\theta}}} \approx \frac{\partial Z\left(\boldsymbol{\theta}, f_{p}, \boldsymbol{\epsilon}=0\right)}{\partial \boldsymbol{\theta}}\right|_{\boldsymbol{\theta}=\overline{\boldsymbol{\theta}}} \\
+\left.\frac{1}{2} \frac{\partial^{2} Z\left(\boldsymbol{\theta}, f_{p}, \boldsymbol{\epsilon}=0\right)}{\partial \boldsymbol{\theta}^{2}}\right|_{\boldsymbol{\theta}=\overline{\boldsymbol{\theta}}} \delta \boldsymbol{\theta} .
\end{aligned}
$$

If we plug in the last two definitions in the (C.6), shorten the notation, and retain only the terms of first order, we will get

$$
\begin{aligned}
0= & \left.\frac{\partial \mathbb{S}(\boldsymbol{\theta}, \overline{\boldsymbol{\theta}})}{\partial \boldsymbol{\theta}}\right|_{\boldsymbol{\theta}=\hat{\boldsymbol{\theta}}} \approx \sum_{p=1}^{P}\left(\frac{\partial Z\left(\overline{\boldsymbol{\theta}}, f_{p}, \boldsymbol{\epsilon}=0\right)}{\partial \boldsymbol{\theta}}\right)^{*} \\
& \times\left(\frac{\partial Z\left(\overline{\boldsymbol{\theta}}, f_{p}, \boldsymbol{\epsilon}=0\right)^{T}}{\partial \boldsymbol{\theta}} \delta \boldsymbol{\theta}-\frac{\partial Z\left(\overline{\boldsymbol{\theta}}, f_{p}, \boldsymbol{\epsilon}=0\right)^{T}}{\partial \boldsymbol{\epsilon}} \boldsymbol{\epsilon}_{p}\right) \\
& +\sum_{p=1}^{P}\left(\frac{\partial Z\left(\overline{\boldsymbol{\theta}}, f_{p}, \boldsymbol{\epsilon}=0\right)^{T}}{\partial \boldsymbol{\theta}} \delta \boldsymbol{\theta}-\frac{\partial Z\left(\overline{\boldsymbol{\theta}}, f_{p}, \boldsymbol{\epsilon}=0\right)^{T}}{\partial \boldsymbol{\epsilon}} \boldsymbol{\epsilon}_{p}\right)^{*} \\
& \times \frac{\partial Z\left(\overline{\boldsymbol{\theta}}, f_{p}, \boldsymbol{\epsilon}=0\right)}{\partial \boldsymbol{\theta}} .
\end{aligned}
$$

If we define

$$
\begin{aligned}
\mathbf{A}_{\theta \theta}\left(f_{p}\right)= & \left(\frac{\partial Z\left(\overline{\boldsymbol{\theta}}, f_{p}, \boldsymbol{\epsilon}=0\right)}{\partial \boldsymbol{\theta}}\right)^{*}\left(\frac{\partial Z\left(\overline{\boldsymbol{\theta}}, f_{p}, \boldsymbol{\epsilon}=0\right)}{\partial \boldsymbol{\theta}}\right)^{T} \\
& +\frac{\partial Z\left(\overline{\boldsymbol{\theta}}, f_{p}, \boldsymbol{\epsilon}=0\right)}{\partial \boldsymbol{\theta}}\left(\left(\frac{\partial Z\left(\overline{\boldsymbol{\theta}}, f_{p}, \boldsymbol{\epsilon}=0\right)}{\partial \boldsymbol{\theta}}\right)^{*}\right)^{T},
\end{aligned}
$$

and

$$
\begin{aligned}
\mathbf{A}_{\theta \epsilon}\left(f_{p}\right)= & \left(\frac{\partial Z\left(\overline{\boldsymbol{\theta}}, f_{p}, \boldsymbol{\epsilon}=0\right)}{\partial \boldsymbol{\theta}}\right)^{*}\left(\frac{\partial Z\left(\overline{\boldsymbol{\theta}}, f_{p}, \boldsymbol{\epsilon}=0\right)}{\partial \boldsymbol{\epsilon}}\right)^{T} \\
& +\frac{\partial Z\left(\overline{\boldsymbol{\theta}}, f_{p}, \boldsymbol{\epsilon}=0\right)}{\partial \boldsymbol{\theta}}\left(\left(\frac{\partial Z\left(\overline{\boldsymbol{\theta}}, f_{p}, \boldsymbol{\epsilon}=0\right)}{\partial \boldsymbol{\epsilon}}\right)^{*}\right)^{T},
\end{aligned}
$$


(note that $\mathbf{A}_{\theta \theta}^{*}\left(f_{p}\right)=\mathbf{A}_{\theta}\left(f_{p}\right)$ and $\mathbf{A}_{\theta \epsilon}^{*}\left(f_{p}\right)=\mathbf{A}_{\theta \epsilon}\left(f_{p}\right)$ ), then we can easily group the various terms of (C.6) as follows

$0=\frac{\partial \mathbb{S}(\boldsymbol{\theta}=\hat{\boldsymbol{\theta}}, \overline{\boldsymbol{\theta}})}{\partial \boldsymbol{\theta}} \approx \sum_{p=1}^{P} \mathbf{A}_{\theta}\left(f_{p}\right) \delta \boldsymbol{\theta}-\sum_{p=1}^{P} \mathbf{A}_{\theta \epsilon}\left(f_{p}\right) \boldsymbol{\epsilon}_{p}$.

If we define

$\mathbf{A}_{\theta}=\sum_{p=1}^{P} \mathbf{A}_{\theta \theta}\left(f_{p}\right)$

and suppose that $\operatorname{det}\left(\mathbf{A}_{\theta}\right) \neq 0$, then the $\delta \boldsymbol{\theta}$ is given by:

$\delta \boldsymbol{\theta}=\mathbf{A}_{\theta}^{-1} \sum_{p=1}^{P} \mathbf{A}_{\theta \epsilon}\left(f_{p}\right) \boldsymbol{\epsilon}_{p}$.

It is trivial to note that if the $\epsilon_{p}$ are normal and independent, then $0=\mathbb{E}[\delta \boldsymbol{\theta}]=\mathbb{E}[\hat{\boldsymbol{\theta}}]-\mathbb{E}[\overline{\boldsymbol{\theta}}]=\mathbb{E}[\hat{\boldsymbol{\theta}}]-\overline{\boldsymbol{\theta}}$ and the asymptotic $\delta \boldsymbol{\theta}$ is Gaussian as well. The covariance of $\delta \boldsymbol{\theta}$ is given by the expected value of the following quantity:

$$
\begin{aligned}
\delta \boldsymbol{\theta} \delta \boldsymbol{\theta}^{T} & =\mathbf{A}_{\theta}^{-1} \sum_{q=1}^{P} \mathbf{A}_{\theta \epsilon}\left(f_{q}\right) \boldsymbol{\epsilon}_{q}\left(\sum_{p=1}^{P} \mathbf{A}_{\theta \epsilon}\left(f_{p}\right) \boldsymbol{\epsilon}_{p}\right)^{T}\left(\mathbf{A}_{\theta}^{-1}\right)^{T}, \\
& =\mathbf{A}_{\theta}^{-1}\left(\sum_{q=1}^{P} \sum_{p=1}^{P} \mathbf{A}_{\theta \epsilon}\left(f_{q}\right) \boldsymbol{\epsilon}_{q} \boldsymbol{\epsilon}_{p}^{T} \mathbf{A}_{\theta \epsilon}\left(f_{p}\right)^{T}\right)\left(\mathbf{A}_{\theta}^{-1}\right)^{T} .
\end{aligned}
$$

Since the $\boldsymbol{\epsilon}$ are independent, then $\mathbb{E}\left[\boldsymbol{\epsilon}_{q} \boldsymbol{\epsilon}_{p}{ }^{T}\right]=\delta_{h p} \boldsymbol{\Sigma}_{\epsilon}$ (the $\delta_{h p}$ is Kronecker's delta), which in turn gives that the asymptotic covariance matrix $\mathbf{V}_{\text {asympt }}$ is given by

$\mathbf{V}_{\text {asympt }}=\mathbb{E}\left[\delta \boldsymbol{\theta} \delta \boldsymbol{\theta}^{T}\right]=\mathbf{A}_{\theta}^{-1} \mathbf{A}_{\epsilon}\left(\mathbf{A}_{\theta}^{-1}\right)^{T}$,

where the $\mathbf{A}_{\epsilon}$ is given by

$\mathbf{A}_{\epsilon}=\sum_{p=1}^{P} \mathbf{A}_{\theta \epsilon}\left(f_{p}\right) \boldsymbol{\Sigma}_{\epsilon} \mathbf{A}_{\theta \epsilon}\left(f_{p}\right)^{T}$.

In our case we note that

$$
\frac{\partial Z}{\partial \boldsymbol{\epsilon}}\left(\boldsymbol{\theta}, f_{p}, \boldsymbol{\epsilon}=0\right)=\left(\begin{array}{c}
Z\left(\boldsymbol{\theta}, f_{p}, \boldsymbol{\epsilon}=0\right) \\
\operatorname{iarg}\left(Z\left(\boldsymbol{\theta}, f_{p}, \boldsymbol{\epsilon}=0\right)\right) Z\left(\boldsymbol{\theta}, f_{p}, \boldsymbol{\epsilon}=0\right)
\end{array}\right),
$$

and that

$\boldsymbol{\Sigma}_{\epsilon}=\left(\begin{array}{cc}\sigma_{A}^{2} & 0 \\ 0 & \sigma_{\phi}^{2}\end{array}\right)$.

The derivation of the asymptotic covariance vas obtained in the case $\overline{\boldsymbol{\theta}}$ is known and equal to the a vector of ones. However in typical experimental situations $\overline{\boldsymbol{\theta}}$ is unknown but it can be estimated sequentially. If applied to the experiment, the asymptotic covariance matrix is then an approximation of the posterior covariance matrix defined by:

$\mathbf{V}_{\text {asympt }} \approx \mathbb{E}\left[\left(\hat{\boldsymbol{\theta}}-\boldsymbol{\theta}_{0}\right)\left(\hat{\boldsymbol{\theta}}-\boldsymbol{\theta}_{0}\right)^{T} \mid \boldsymbol{\theta}_{0}=\overline{\boldsymbol{\theta}}\right]$.

In the sequential case we have set the value around which we compute the $\mathbf{V}_{\text {asympt }}$ to be the $M$-th realization of the estimated parameter vector, i.e., $\boldsymbol{\theta}_{0}=\hat{\boldsymbol{\theta}}_{M}$.

\section{Appendix D. Computational details}

The computations in this paper were run both in Matlab [72] and Octave [73].

\section{D.1. Data fitting}

The data fitting procedure aims at determining an estimate of the parameters by minimizing the following functional of $\boldsymbol{\theta}$

$\mathbb{S}(\boldsymbol{\theta})=\sum_{j=1}^{P}\left|Z\left(\boldsymbol{\theta}, f_{j}\right)-Z_{\text {meas }} \cdot\left(\boldsymbol{\theta}, f_{j}\right)\right|^{2}$

We performed this minimization in both Matlab and Octave and the $\hat{\boldsymbol{\theta}}=\operatorname{argmin}_{\boldsymbol{\theta}} \mathbb{S}(\boldsymbol{\theta})$ was achieved by running an SQP algorithm in both Matlab and Octave [74], and an interior point algorithm with Matlab [75-77] with box constraints $0.1 \leq \theta_{i} \leq 10$. Both algorithms were shown to converge to the same minimizer for all case.

\section{D.2. Optimization strategy in $O E D$}

In both the Matlab and Octave implementations, we defined two functions, one that computes the asymptotic covariance matrix $\mathbf{V}_{\text {asympt }}$ given in Section Appendix C, the other one that computes the experimental time $t_{\exp }$ given in Section 4.2. In Matlab we utilized the optimization procedure fmincon, which finds minimum of either $\mathbf{V}_{\text {asympt }}$ and $t_{\text {exp }}$, allowing both box and nonlinear constraints; we used the interior-point algorithm to minimize the constrained objective function . In Octave we utilized a custommade SQP algorithm.

We stress that fmincon searches for a local minimum. A global minimization strategy in the case of a large $\xi$ vector would in fact require a large amount of resources. We however checked if the searched minimum is global by randomizing the initial $\xi=\mathbf{f}$ within the box constraint bounds. We discovered that multiple local minima can be found and that the solution is sensitive to the starting optimization point and the optimizer's options. Hence, we ensured the convergence to a minimum by restarting the optimized at least 20 times for each minimization.

\section{References}

[1] D.D. Macdonald, Electrochim. Acta 51 (2006) 1376.

[2] J.R. Macdonald, Ann. Biomed. Eng. 20 (1992) 289.

[3] B.-Y. Chang, S.-M. Park, Ann. Rev. Anal. Chem. 3 (2010) 207.

[4] E. Barsoukov, J.R. Macdonald (Eds.), Impedance Spectroscopy: Theory, Experiment, and Applications, Wiley and Sons, New York, 2005.

[5] B. Pejcic, R. De Marco, Electrochim. Acta 51 (2006) 6217.

[6] Q. Wang, J.-E. Moser, M. Grätzel, J. Phys. Chem. B 109 (2005) 14945.

[7] K. Jättner, Electrochim. Acta 35 (1990) 1501.

[8] R. Gómez, R. Bashir, A. Sarikaya, M.R. Ladisch, J. Sturgis, J.P. Robinson, T. Geng, A.K. Bhunia, H.L. Apple, S. Wereley, Biomed. Microdev. 3 (2001) 201.

[9] M.W. Louie, A. Hightower, S.M. Haile, ACS Nano 4 (2010) 2811.

[10] M.E. Orazem, P. Agarwal, A.N. Jansen, P.T. Wojcik, L.H. Garcia-Rubio, Electrochim. Acta 38 (1993) 1903.

[11] J. Fleig, J. Electroceram. 13 (2004) 637.

[12] D. Dees, E. Gunen, D. Abraham, A. Jansen, J. Prakash, J. Electrochem. Soc. 152 (2005) A1409.

[13] Q.-A. Huang, R. Hui, B. Wang, J. Zhang, Electrochim. Acta 52 (2007) 8144.

[14] B.A. Boukamp, Solid State Ionics 18-19 (1986) 136.

[15] J.R. Macdonald, L.D. Potter Jr., Solid State Ionics 24 (1987) 61

[16] J.R. Macdonald, CNLS (Complex Nonlinear Least Squares) lmmittance Fitting Program LEVM Manual, Houston, TX, Version 7.11 Edition, 1999.

[17] A. Atkinson, A. Donev, Optimum Experimental Design, Oxford Statistical Science Series, Clarendon Press, 1992.

[18] R.H. Milocco, E.B. Castro, S.G. Real, Electrochim. Acta 47 (2002) 2035

[19] M.E. Huerta-Garrido, M.D. Pritzker, J. Electroanal. Chem. 594 (2006) 118.

[20] F. Berthier, J.P. Diard, P. Landaud, C. Montella, J. Electroanal. Chem. 362 (1993) 13.

[21] J. Fleig, J. Electroceram. 13 (2004) 637

[22] A.C. Atkinson, D.R. Cox, J. R. Stat. Soc. Ser. B (Methodological) 36 (1974) 321.

[23] G.E.P. Box, W.J. Hill, Technometrics 9 (1967) 57.

[24] P.D.H. Hill, Technometrics 20 (1978) 15.

[25] F. Pukelsheim, Optimal Design of Experiments, Wiley Series in Probability and Mathematical Statistics, John Wiley \& Sons, 1993.

[26] A. Pázman, Foundations of Optimum Experimental Design. Transl. from the Slovakian by the author, D. Reidel Publishing Company, 1986.

[27] V.V. Fedorov, P. Hackl, Model-oriented Design of Experiments. Lecture Notes in Statistics, Springer, New York, NY, 1997. 
[28] L. Pronzato, E. Walter, Identification of Parametric Models from Experimental Data, Springer, Heidelberg, 1997.

[29] A. Bardow, W. Marquardt, V. Göke, H. Koss, K. Lucas, AIChE J. 49 (2003) 323.

[30] S. Körkel, E. Kostina, H. Bock, J. Schlöder, Optim. Methods Softw. 19 (2004) 327.

[31] D. Ucinski, Optimal Measurement Methods for Distributed Parameter System Identification, CRC Press, 2005.

[32] E. Balsa-Canto, A. Alonso, J. Banga, Syst. Biol., IET 2 (2008) 163.

[33] T. Carraro, V. Heuveline, R. Rannacher, Reactive Flows, Diffusion, Transport, Springer, Berlin Heidelberg, 2007, pp. 211-249.

[34] D. Cox, Principles of Statistical Inference, Cambridge University Press, 2006.

[35] L. Wasserman, All of Statistics: A Concise Course in Statistical Inference, Springer, 2003.

[36] E. Marchand, F. Clément, J. Roberts, G. Pépin, Adv. Water Resour. 31 (2008) 1025 .

[37] I. Bauer, H. Bock, S. Körkel, J. Schlöder, J. Comp. Appl. Math. 120 (2000) 1.

[38] H.M. Bücker, G. Corliss, P. Hovland (Eds.), Automatic Differentiation: Applications, Theory, and Implementations, Springer-Verlag, New York, NY, USA, 2009.

[39] J. Jamnik, J. Maier, Phys. Chem. Chem. Phys. 3 (2001) 1668

[40] W. Lai, S. Haile, J. Am. Ceram. Soc. 88 (2005) 2979

[41] F. Ciucci, Y. Hao, D.G. Goodwin, Phys. Chem. Chem. Phys. 11 (2009) 11243.

[42] W.C. Chueh, W. Lai, S.M. Haile, Solid State Ionics 179 (2008) 1036.

[43] J. Jamnik, J. Maier, S. Pejovnik, Electrochim. Acta 44 (1999) 4139.

[44] F. Ciucci, Continuum Modeling of Mixed Conductors: A Study of Ceria, Ph.D. thesis, California Institute of Technology, 2009.

[45] F. Ciucci, W.C. Chueh, D.G. Goodwin, S.M. Haile, Phys. Chem. Chem. Phys. 13 (2011) 2121.

[46] M.E. Orazem, T. El Moustafid, C. Deslouis, B. Tribollet, J. Electrochem. Soc. 143 (1996) 3880.

[47] M.E. Orazem, P. Shukla, M.A. Membrino, Electrochim. Acta 47 (2002) 2027.

[48] M.E. Orazem, J. Electroanal. Chem. 572 (2004) 317.

[49] P.K. Shukla, M.E. Orazem, O.D. Crisalle, Electrochim. Acta 49 (2004) 2881.

[50] S.L. Carson, M.E. Orazem, O.D. Crisalle, L. Garcia-Rubio, J. Electrochem. Soc. 150 (2003) 477.

[51] J.R. Dygas, M.W. Breiter, Electrochim. Acta 44 (1999) 4163.
[52] P. Zoltowski, J. Electroanal. Chem. 375 (1994) 45.

[53] P. Zoltowski, J. Electroanal. Chem. 178 (1984) 11.

[54] P. Zoltowski, J. Electroanal. Chem. 424 (1997) 173.

[55] J.R. Macdonald, D.R. Franceschetti, J. Electroanal. Chem. 307 (1991) 1.

[56] T. Dickinson, R. Whitfield, Electrochim. Acta 22 (1977) 385.

[57] V. Horvat-Radosevic, K. Kvastek, M. Kraljic-Rokovic, Electrochim. Acta 51 (2006) 3417.

[58] T. Hilpert, Solid State Ionics 177 (2006) 1577.

[59] J.R. Macdonald, Impedance Spectroscopy-Emphasizing Solid Materials and Systems, Wiley-Interscience, John Wiley and Sons, 1987.

[60] J.R. Macdonald, W.J. Thompson, Commun. Stat. Simulat. 20 (1991) 843.

[61] G. Seber, C. Wild, Nonlinear Regression. Wiley Series in Probability and Mathematical Statistics, John Wiley \& Sons Ltd., 1989.

[62] D.M. Bates, D.G. Watts, Nonlinear Regression Analysis and its Applications, Wiley, 1988.

[63] Solartron, http://www.solartronanalytical.com/pages/1260afrapage.htm, 2011.

[64] C.-F. Wu, Biometrika 72 (1985) 553.

[65] H.P. Wynn, Ann. Math. Stat. 41 (1970) 1655.

[66] G. Buzzi-Ferraris, P. Forzatti, G. Emig, H. Hofmann, Chem. Eng. Sci. 39 (1984) 81.

67] A.K. Agarwal, M.L. Brisk, Ind. Eng. Chem. 24 (1985) 203.

[68] K.W. Vugrin, L.P. Swiler, R.M. Roberts, A.M. Roberts, N.J. Stucky-mack, S.P. Sullivan, Confidence Region Estimation Techniques for Nonlinear Regression: Three Case Studies, SANDIA REPORT - SAND2005-6893, 2005.

[69] J.B. Ramsey, Econometrica 46 (July) (1978) 901.

[70] C.-F. Wu, Ann. Stat. 9 (May) (1981) 501.

[71] T. Amemiya, Non-linear Regression Models, vol. 1, Elsevier, pp. 333-389.

[72] MATLAB Version 7.1, The MathWorks Inc., Natick, MA, 2009.

[73] J.W. Eaton, GNU Octave Manual, Network Theory Limited, 2002.

[74] P.E. Gill, W. Murray, M.A. Saunders, SIAM J. Optim. 12 (2002) 979.

[75] T.F. Coleman, A. Verma, Comp. Optim. Appl. 20 (2001) 61.

[76] R.H. Byrd, J.C. Gilbert, J. Nocedal, Math. Prog. 89 (2000) 149

[77] R.A. Waltz, J.L. Morales, J. Nocedal, D. Orban, Math. Prog. 107 (2006) 391. 